\title{
Microwave- and Photoirradiation-Induced Staudinger Reactions of Cyclic Imines and Ketenes Generated from $\alpha$-Diazoketones. A Further Investigation into the Stereochemical Process
}

\author{
Yong Liang, Lei Jiao, Shiwei Zhang, and Jiaxi $\mathrm{Xu}^{*}$ \\ Key Laboratory of Bioorganic Chemistry and Molecular Engineering of Ministry of Education, Department \\ of Chemical Biology, College of Chemistry and Molecular Engineering, Peking University, Beijing 100871, \\ P. R. China
}

\section{Supplemental Materials}

Content S1

Experimental Details. S2

The spectroscopic data of compounds $5 \mathbf{a}-\mathbf{c}, \mathbf{6 b}-\mathbf{d}, 7 \mathbf{b}, \mathbf{8}, 9 \mathbf{a}-\mathbf{d}, 10 a-b$, and 11 S3

Copies of ${ }^{1} \mathrm{H}$ NMR and ${ }^{13} \mathrm{C}$ NMR spectra of all the unknown compounds S9

Copies of DEPT and gHSQC spectra of the unknown compound $7 \mathbf{a}$ S17

Copies of DEPT and gHSQC spectra of the unknown compound 9a..... S21

Copy of DEPT spectrum of the unknown compound 10a S26

Crystal structure of $\mathbf{6 a}$ S29 


\section{Experimental Details}

General. $\alpha$-Diazoketone $1,{ }^{1}$ imines $\mathbf{2 a},{ }^{2} \mathbf{2 b}-\mathbf{c},{ }^{3} \mathbf{3 a}-\mathbf{e},{ }^{4}$ and $\mathbf{4 a}-\mathbf{b}^{5}$ were prepared according to published procedures. Dichloromethane and 1,2-dichlorobenzene were refluxed with $\mathrm{CaH}_{2}$ and freshly distilled prior to use. All reactions were performed under a nitrogen atmosphere. For microwave assisted reactions, a domestic microwave oven was employed and the power of microwave irradiation was set to about $500 \mathrm{~W}$ (Medium). Nitrogen inlet and outlet were connected via a reflux condenser with the flask in the microwave oven. Photochemical reactions were carried out in a quartz tube equipped with a cooling finger, nitrogen inlet and outlet. The reaction mixture was cooled by a circulation of $-20{ }^{\circ} \mathrm{C}$ liquid though the cooling finger. A $500 \mathrm{~W}$ high-pressure mercury lamp was used as UV source, with a distance of 10 $\mathrm{cm}$ to the quartz tube (shown in the following figure).

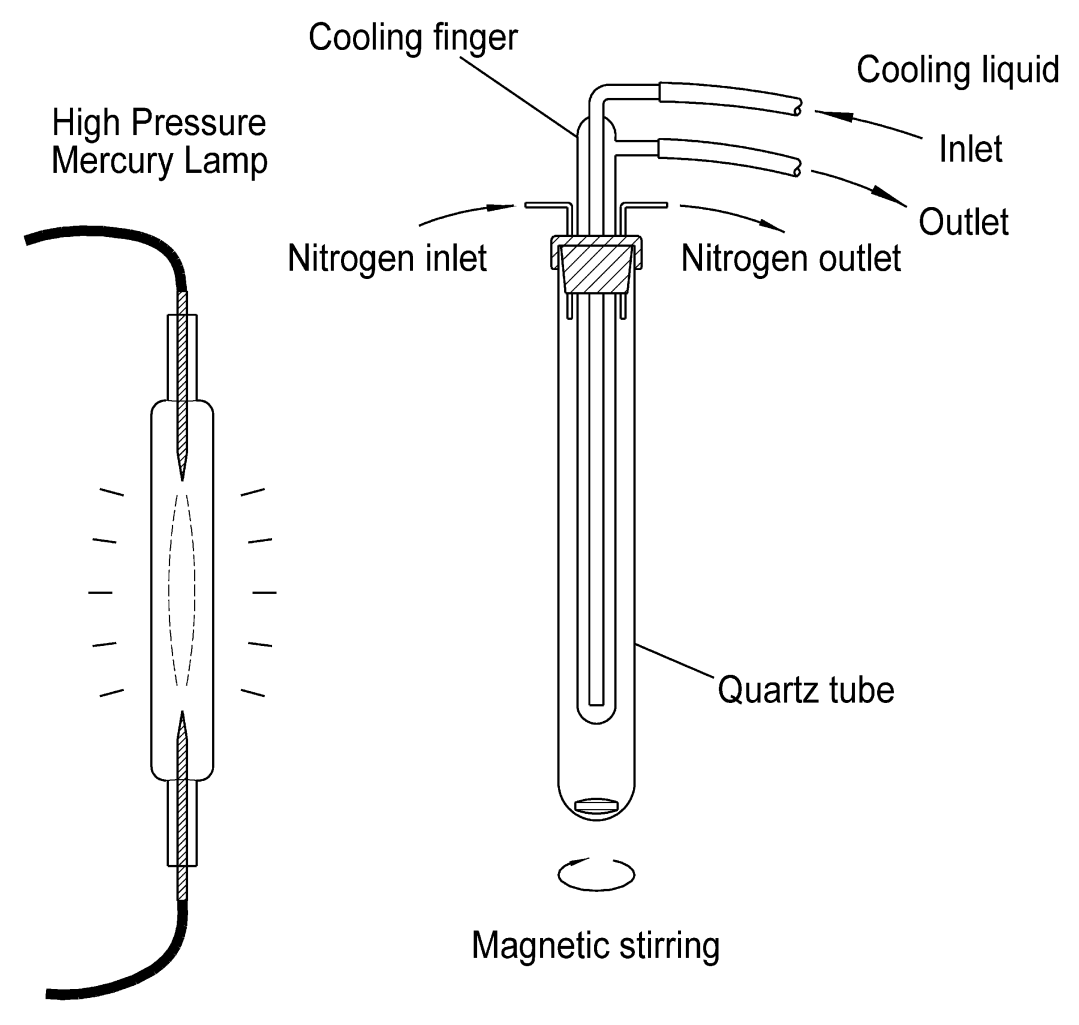

\footnotetext{
${ }^{1}$ Podlech, J.; Seebach, D. Liebigs Ann., 1995, 1217-1228.

2 Bigelow, L. A.; Eatough, H. Org. Syn., Coll. Vol. 1, 80.

${ }^{3}$ Linder, M. R.; Frey, W. U.; Podlech, J. J. Chem. Soc., Perkin Trans. 1 2001, 2566-2577.

${ }^{4}$ Wenker, H. J. Am. Chem. Soc., 1935, 57, 6, 1079-1080.

${ }^{5}$ Wardrop, A. W. H.; Sainsbury, G. L.; Harrison, J. M.; Inch, T. D. J. Chem. Soc., Perkin Trans. 1, 1976, $1279-1285$
} 
( \pm )-trans $-3-$ Benzyloxycarbonylaminomethyl-1,4-diphenylazetidin-2-one (5a)<smiles>O=C(NC[C@H]1C(=O)N(c2ccccc2)C1c1ccccc1)c1ccccc1</smiles>

White solid; m.p. $138-139{ }^{\circ} \mathrm{C} ;{ }^{1} \mathrm{H}$ NMR (300 MHz): $\delta 3.21$ (dt, $\left.J=2.4,5.7 \mathrm{~Hz}, 1 \mathrm{H}\right), 3.72(\mathrm{t}, J$ $=5.7 \mathrm{~Hz}, 2 \mathrm{H}), 4.93(\mathrm{~s}, 1 \mathrm{H}), 5.10(\mathrm{~d}, J=12.3 \mathrm{~Hz}, 1 \mathrm{H}), 5.15(\mathrm{~d}, J=12.3 \mathrm{~Hz}, 1 \mathrm{H}), 5.31$ (s, br, 1H), $7.06(\mathrm{~m}, 1 \mathrm{H}), 7.23-7.32(\mathrm{~m}, 14 \mathrm{H}) .{ }^{13} \mathrm{C} \mathrm{NMR}(75.5 \mathrm{MHz}): \delta 38.7,58.5,60.5,66.9,117.1$, $124.0,125.8,128.0,128.1,128.4,128.5,129.0,129.1,136.2,137.2,156.8,165.4$. MS (EI) m/z: $386\left(\mathrm{M}^{+}, 4.5\right), 358\left(\mathrm{M}^{+}-\mathrm{CO}, 8.0\right), 295\left(\mathrm{M}^{+}-\mathrm{Bn}, 12\right), 278\left(\mathrm{M}^{+}-\mathrm{BnOH}, 5.5\right), 222$ $\left(\mathrm{M}^{+}-\mathrm{CbzNHCH}_{2}, 16\right), 91\left(\mathrm{Bn}^{+}, 100\right)$; IR $v\left(\mathrm{~cm}^{-1}\right)$ : 1748, 1720. Calcd for $\mathrm{C}_{24} \mathrm{H}_{22} \mathrm{~N}_{2} \mathrm{O}_{3}$ : 386.1630. Found: 386.1640 .

( \pm - trans $^{-3}$-Benzyloxycarbonylaminomethyl-1-benzyl-4-phenylazetidin-2-one (5b)<smiles>O=C(NCC1C(=O)C(c2ccccc2)C1c1ccccc1)OCc1ccccc1</smiles>

White solid; m.p. $82-83{ }^{\circ} \mathrm{C} ;{ }^{1} \mathrm{H}$ NMR (300 MHz): $\delta 3.15$ (m, 1H), $3.61(\mathrm{~m}, 2 \mathrm{H}), 3.74(\mathrm{~d}, J=$ $15.0 \mathrm{~Hz}, 1 \mathrm{H}), 4.29$ (d, $J=1.5 \mathrm{~Hz}, 1 \mathrm{H}), 4.82(\mathrm{~d}, J=15.0 \mathrm{~Hz}, 1 \mathrm{H}), 4.97$ (d, $J=12.3 \mathrm{~Hz}, 1 \mathrm{H})$, $5.10(\mathrm{~d}, J=12.3 \mathrm{~Hz}, 1 \mathrm{H}), 5.34(\mathrm{~s}, \mathrm{br}, 1 \mathrm{H}), 7.10-7.38(\mathrm{~m}, 15 \mathrm{H}) .{ }^{13} \mathrm{C}$ NMR $(75.5 \mathrm{MHz}): \delta 38.5$, 44.4, 57.6, 60.3, 66.7, 126.4, 127.6, 127.9, 128.0, 128.2, 128.4, 128.7, 128.9, 135.2, 136.2, 136.9, 156.6, 168.1. MS (EI) m/z: $400\left(\mathrm{M}^{+}, 0.3\right), 372\left(\mathrm{M}^{+}-\mathrm{CO}, 1.2\right), 309\left(\mathrm{M}^{+}-\mathrm{Bn}, 5.3\right), 236$ $\left(\mathrm{M}^{+}-\mathrm{CbzNHCH}_{2}, 0.9\right), 91\left(\mathrm{Bn}^{+}, 100\right)$; IR $v\left(\mathrm{~cm}^{-1}\right)$ : 1727, 1711. Calcd for $\mathrm{C}_{25} \mathrm{H}_{24} \mathrm{~N}_{2} \mathrm{O}_{3}$ : 400.1787. Found: 400.1783.

( \pm )-trans $-3-$ Benzyloxycarbonylaminomethyl-1-tertbutyl-4-phenylazetidin-2-one (5c)<smiles>O=C(NC[C@@H]1C(=O)N(Cc2ccccc2)C1c1ccccc1)c1ccccc1</smiles>

White solid; m.p. $146-147{ }^{\circ} \mathrm{C} ;{ }^{1} \mathrm{H}$ NMR (300 MHz): $\delta 1.20$ (s, 9H), 2.90 (dt, $J=1.8,5.6 \mathrm{~Hz}$, $1 \mathrm{H}), 3.60(\mathrm{~m}, 2 \mathrm{H}), 4.44(\mathrm{~d}, J=1.8 \mathrm{~Hz}, 1 \mathrm{H}), 5.12(\mathrm{~d}, J=12.3 \mathrm{~Hz}, 1 \mathrm{H}), 5.16(\mathrm{~d}, J=12.3 \mathrm{~Hz}$, 1H), 5.21 (s, br, 1H), 7.31-7.36 (m, 10H). ${ }^{13} \mathrm{C}$ NMR (75.5 MHz): $\delta$ 28.1, 38.6, 54.7, 57.4, 58.9, 66.8, 126.2, 128.1, 128.5, 128.7, 136.4, 140.2, 156.7, 168.5. MS (EI) m/z: $366\left(\mathrm{M}^{+}, 1.1\right)$, $338\left(\mathrm{M}^{+}-\mathrm{CO}, 6.6\right), 275\left(\mathrm{M}^{+}-\mathrm{Bn}, 3.9\right), 91\left(\mathrm{Bn}^{+}, 73\right)$; IR $v\left(\mathrm{~cm}^{-1}\right): 1721,1706$. Calcd for $\mathrm{C}_{22} \mathrm{H}_{26} \mathrm{~N}_{2} \mathrm{O}_{3}: 366.1943$. Found: 366.1935. 


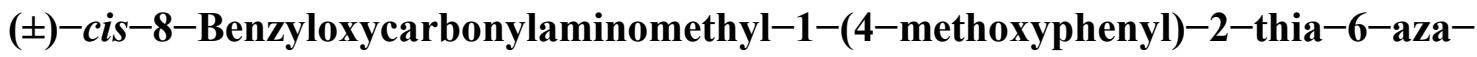
bicyclo[4.2.0] octan-7-one (6b)<smiles>[2H][Pb]1(OC)CCCN2C(=O)[C@H](CNC(=O)OCc3ccccc3)[C@]21P</smiles>

Colorless oil; ${ }^{1} \mathrm{H}$ NMR (300 MHz): $\delta 1.81(\mathrm{~m}, 2 \mathrm{H}), 2.62(\mathrm{~m}, 2 \mathrm{H}), 3.05(\mathrm{~m}, 3 \mathrm{H}), 3.68(\mathrm{t}, J=$ $7.8 \mathrm{~Hz}, 1 \mathrm{H}), 3.82(\mathrm{~s}, 3 \mathrm{H}), 4.09(\mathrm{~m}, 1 \mathrm{H}), 4.74(\mathrm{~s}, \mathrm{br}, 1 \mathrm{H}), 5.02(\mathrm{~s}, 2 \mathrm{H}), 6.92(\mathrm{~d}, J=8.5 \mathrm{~Hz}, 2 \mathrm{H})$, 7.32-7.35 (m, 5H), $7.46(\mathrm{~d}, J=8.5 \mathrm{~Hz}, 2 \mathrm{H}) .{ }^{13} \mathrm{C} \mathrm{NMR}(75.5 \mathrm{MHz}): \delta 23.8,25.4,37.4,37.9$, $55.3,64.3,65.4,66.6,114.0,128.0,128.4,128.9,136.3,155.8,159.5,166.4$. MS (EI) m/z: $412\left(\mathrm{M}^{+}, 0.70\right), 321\left(\mathrm{M}^{+}-\mathrm{Bn}, 1.1\right), 304\left(\mathrm{M}^{+}-\mathrm{BnOH}, 3.1\right), 248\left(\mathrm{M}^{+}-\mathrm{CbzNHCH}_{2} \mathrm{O}, 100\right)$; IR $v$ $\left(\mathrm{cm}^{-1}\right)$ : 1750,1717 . Calcd for $\mathrm{C}_{22} \mathrm{H}_{24} \mathrm{~N}_{2} \mathrm{O}_{4} \mathrm{~S}: 412.1457$. Found: 412.1439 .

( \pm )-cis-8-Benzyloxycarbonylaminomethyl-1-(4-methylphenyl)-2-thia-6-aza-bicyclo [4.2.0]octan-7-one (6c)<smiles>O=C(NC[C@H]1C(=O)N2CCCS[C@]12P)OCc1ccccc1</smiles>

Colorless oil; ${ }^{1} \mathrm{H}$ NMR (300 MHz): $\delta 1.81$ (m, 2H), 2.36 (s, 3H), 2.61 (m, 2H), 3.04 (m, 3H), $3.69(\mathrm{t}, J=8.0 \mathrm{~Hz}, 1 \mathrm{H}), 4.10(\mathrm{~m}, 1 \mathrm{H}), 4.76(\mathrm{~s}, \mathrm{br}, 1 \mathrm{H}), 5.02(\mathrm{~s}, 2 \mathrm{H}), 7.19-7.43(\mathrm{~m}, 9 \mathrm{H}) .{ }^{13} \mathrm{C}$ NMR (75.5 MHz): $\delta 21.0,23.7,25.3,37.5,37.8,64.1,65.5,66.6,127.4,128.0,128.4,129.4$, 133.7, 136.3, 138.3, 155.7, 166.3. MS (EI) m/z: $396\left(\mathrm{M}^{+}, 0.64\right), 368\left(\mathrm{M}^{+}-\mathrm{CO}, 0.76\right), 305$ $\left(\mathrm{M}^{+}-\mathrm{Bn}, 0.5\right), 288\left(\mathrm{M}^{+}-\mathrm{BnOH}, 5.3\right), 232\left(\mathrm{M}^{+}-\mathrm{CbzNHCH}_{2}, 100\right)$; IR $v\left(\mathrm{~cm}^{-1}\right): 1756,1717$ Calcd for $\mathrm{C}_{22} \mathrm{H}_{24} \mathrm{~N}_{2} \mathrm{O}_{3} \mathrm{~S}$ : 396.1508. Found: 396.1491.

( \pm )-cis-8-Benzyloxycarbonylaminomethyl-1-(4-chlorophenyl)-2-thia-6-aza-bicyclo [4.2.0]octan-7-one (6d)

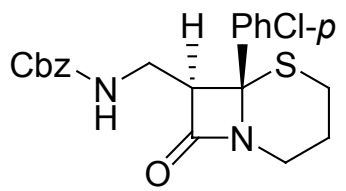

Colorless oil; ${ }^{1} \mathrm{H}$ NMR (300 MHz): $\delta 1.81(\mathrm{~m}, 2 \mathrm{H}), 2.59(\mathrm{~m}, 2 \mathrm{H}), 3.00(\mathrm{~m}, 3 \mathrm{H}), 3.71(\mathrm{t}, J=$ $7.8 \mathrm{~Hz}, 1 \mathrm{H}), 4.10(\mathrm{~m}, 1 \mathrm{H}), 4.90(\mathrm{~s}, \mathrm{br}, 1 \mathrm{H}), 5.01(\mathrm{~s}, 2 \mathrm{H}), 7.28-7.49(\mathrm{~m}, 9 \mathrm{H}) .{ }^{13} \mathrm{C}$ NMR $(75.5$ $\mathrm{MHz}): \delta 23.5,25.3,37.5,37.8,64.1,65.0,66.7,128.0,128.4,128.8,129.0,134.4,135.6$, 136.2, 155.7, 166.2. MS (EI) m/z: $416\left(\mathrm{M}^{+}, 0.62\right), 388\left(\mathrm{M}^{+}-\mathrm{CO}, 1.1\right), 325\left(\mathrm{M}^{+}-\mathrm{Bn}, 3.8\right), 308$ $\left(\mathrm{M}^{+}-\mathrm{BnOH}, 4.2\right), 252\left(\mathrm{M}^{+}-\mathrm{CbzNHCH}_{2}, 75\right), 91\left(\mathrm{Bn}^{+}, 100\right)$; IR $v\left(\mathrm{~cm}^{-1}\right): 1752,1717$. Calcd for $\mathrm{C}_{21} \mathrm{H}_{21} \mathrm{ClN}_{2} \mathrm{O}_{3} \mathrm{~S}: 416.0961$. Found: 416.0958. 
$( \pm)-$ trans$^{-2}-$ Benzyloxycarbonylaminomethyl-4-methyl-azeto $[1,2-d]-\operatorname{dibenzo}[b, f]$ oxazepin-1-one (7b)<smiles>Cc1ccc2c(c1)[C@@H]1Oc3ccccc3N2C(=O)C1CNC(=O)OCc1ccccc1</smiles>

Pale yellow oil; ${ }^{1} \mathrm{H}$ NMR (400 MHz): $\delta 2.32(\mathrm{~s}, 3 \mathrm{H}), 3.83(\mathrm{~m}, 3 \mathrm{H}), 5.13(\mathrm{~s}, 2 \mathrm{H}), 5.36$ (s, br, $1 \mathrm{H}), 5.49(\mathrm{~s}, 1 \mathrm{H}), 6.97-7.07(\mathrm{~m}, 3 \mathrm{H}), 7.11(\mathrm{~m}, 2 \mathrm{H}), 7.19-7.22(\mathrm{~m}, 1 \mathrm{H}), 7.29-7.33(\mathrm{~m}, 5 \mathrm{H})$, 7.94-7.96 (m, 1H). ${ }^{13} \mathrm{C}$ NMR (75.5 MHz): $\delta 20.8,38.8,53.8,55.9,66.8,119.8,121.0,121.4$, $124.3,124.9,126.5,127.9,128.0,128.4,129.5,129.7,130.4,134.8,136.1,144.0,155.9$, 156.6, 163.8. MS (EI) m/z: $414\left(\mathrm{M}^{+}, 2.3\right), 323\left(\mathrm{M}^{+}-\mathrm{Bn}, 18\right), 306\left(\mathrm{M}^{+}-\mathrm{BnOH}, 26\right), 250$ $\left(\mathrm{M}^{+}-\mathrm{CbzNHCH}_{2}, 21\right)$; IR $v\left(\mathrm{~cm}^{-1}\right)$ : 1747, 1716. Calcd for $\mathrm{C}_{25} \mathrm{H}_{22} \mathrm{~N}_{2} \mathrm{O}_{4}$ : 414.1580. Found: 414.1587.

( \pm )-1-Benzyloxycarbonyl-2,3-diphenyl-tetrahydropyrimidin-4(1H)-one $(8)$<smiles>CC(C)(C)OC(=O)N1CCC(=O)N(c2ccccc2)C1c1ccccc1</smiles>

Colorless oil; ${ }^{1} \mathrm{H}$ NMR (300 MHz): $\delta 2.58(\mathrm{~m}, 1 \mathrm{H}), 2.68(\mathrm{~m}, 1 \mathrm{H}), 3.44(\mathrm{~m}, 1 \mathrm{H}), 4.04(\mathrm{~m}, 1 \mathrm{H})$, $5.28(\mathrm{~s}, 2 \mathrm{H}), 7.15(\mathrm{~s}, 1 \mathrm{H}), 7.23-7.40(\mathrm{~m}, 15 \mathrm{H}) .{ }^{13} \mathrm{C} \mathrm{NMR}(75.5 \mathrm{MHz}): \delta 31.4,37.7,68.0,72.4$, 125.6, 126.0, 126.7, 128.0, 128.3, 128.5, 128.8, 129.0, 135.7, 138.0, 140.4, 155.2, 168.2. MS (EI) m/z: $386\left(\mathrm{M}^{+}, 3.8\right), 295\left(\mathrm{M}^{+}-\mathrm{Bn}, 61\right), 91\left(\mathrm{Bn}^{+}, 100\right)$; IR $v\left(\mathrm{~cm}^{-1}\right): 1702,1686$. Calcd for $\mathrm{C}_{24} \mathrm{H}_{22} \mathrm{~N}_{2} \mathrm{O}_{3}: 386.1630$. Found: 386.1635 .

( \pm -5-Benzyloxycarbonyl-6-phenyl-1,5-diaza-7-thia-bicyclo[4.4.0]decan-2-one (9a)<smiles>O=C(O)N1CCC(=O)N2CCCSC12c1ccccc1</smiles>

Colorless oil; ${ }^{1} \mathrm{H}$ NMR (300 MHz): $\delta 1.70(\mathrm{~m}, 1 \mathrm{H}), 1.85(\mathrm{~m}, 1 \mathrm{H}), 2.20(\mathrm{~m}, 1 \mathrm{H}), 2.80(\mathrm{~m}, 3 \mathrm{H})$, $3.20(\mathrm{ddd}, J=3.7,12.1,13.2 \mathrm{~Hz}, 1 \mathrm{H}), 3.88(\mathrm{ddd}, J=3.7,10.4,13.2 \mathrm{~Hz}, 1 \mathrm{H}), 4.27$ (dt, $J=$ 13.2, $4.8 \mathrm{~Hz}, 1 \mathrm{H}), 4.53(\mathrm{~m}, 1 \mathrm{H}), 4.86(\mathrm{~s}, 2 \mathrm{H}), 7.07-7.10(\mathrm{~m}, 2 \mathrm{H}), 7.27-7.39(\mathrm{~m}, 6 \mathrm{H})$, 7.65-7.68 (m, 2H). ${ }^{13} \mathrm{C}$ NMR (75.5 MHz): $\delta$ 24.5, 29.8, 33.1, 39.9, 40.0, 67.8, 85.6, 128.1, 128.2, 128.3, 128.4, 135.3, 140.4, 153.3, 167.3. MS (EI) m/z: $382\left(\mathrm{M}^{+}, 12\right), 291\left(\mathrm{M}^{+}-\mathrm{Bn}, 4.8\right)$, 
247 (9.6), 173 (4.2), $91\left(\mathrm{Bn}^{+}, 100\right)$; IR $v\left(\mathrm{~cm}^{-1}\right)$ : 1700, 1651. Calcd for $\mathrm{C}_{21} \mathrm{H}_{22} \mathrm{~N}_{2} \mathrm{O}_{3} \mathrm{~S}$ : 382.1351. Found: 382.1352 .

( \pm )-5-Benzyloxycarbonyl-6-(4-methoxyphenyl)-1,5-diaza-7-thia-bicyclo[4.4.0]decan $-2-$ one $(9 b)$

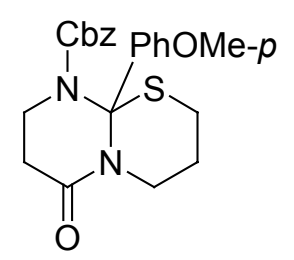

Colorless oil; ${ }^{1} \mathrm{H}$ NMR (300 MHz): $\delta 1.68$ (m, 1H), $1.84(\mathrm{~m}, 1 \mathrm{H}), 2.27(\mathrm{~m}, 1 \mathrm{H}), 2.77(\mathrm{~m}, 3 \mathrm{H})$, 3.17 (ddd, $J=3.6,12.3,13.2 \mathrm{~Hz}, 1 \mathrm{H}), 3.81(\mathrm{~s}, 3 \mathrm{H}), 3.88(\mathrm{~m}, 1 \mathrm{H}), 4.25$ (dt, $J=13.2,4.8 \mathrm{~Hz}$, $1 \mathrm{H}), 4.54(\mathrm{dm}, J=13.8 \mathrm{~Hz}, 1 \mathrm{H}), 4.86(\mathrm{~d}, J=12.0 \mathrm{~Hz}, 1 \mathrm{H}), 4.92(\mathrm{~d}, J=12.0 \mathrm{~Hz}, 1 \mathrm{H}), 6.80$ (d, $J=8.8 \mathrm{~Hz}, 2 \mathrm{H}), 7.11-7.13(\mathrm{~m}, 2 \mathrm{H}), 7.27-7.31(\mathrm{~m}, 3 \mathrm{H}), 7.56(\mathrm{~d}, J=8.8 \mathrm{~Hz}, 2 \mathrm{H}) .{ }^{13} \mathrm{C} \mathrm{NMR}$ (75.5 MHz): $\delta$ 24.6, 29.8, 33.2, 39.9, 40.0, 55.2, 67.8, 85.4, 113.3, 128.1, 128.2, 128.3, 129.6, 132.3, 135.4, 153.3, 159.4, 167.3. MS (EI) m/z: $412\left(\mathrm{M}^{+}, 33\right), 367$ (12), $321\left(\mathrm{M}^{+}-\mathrm{Bn}, 20\right)$, 277 (27), 203 (6.0), 91 (100); IR $v\left(\mathrm{~cm}^{-1}\right)$ : 1702, 1659. Calcd for $\mathrm{C}_{22} \mathrm{H}_{24} \mathrm{~N}_{2} \mathrm{O}_{4} \mathrm{~S}: 412.1457$. Found: 412.1445 .

$( \pm)-5-B e n z y l o x y c a r b o n y l-6-(4-m e t h y l p h e n y l)-1,5-$ diaza-7-thia-bicyclo[4.4.0]decan2-one (9c)<smiles>CCCCN1CCC(=O)N2CCCSC12P(C)(=O)O</smiles>

Colorless oil; ${ }^{1} \mathrm{H}$ NMR (300 MHz): $\delta 1.69$ (m, 1H), $1.83(\mathrm{~m}, 1 \mathrm{H}), 2.24(\mathrm{~m}, 1 \mathrm{H}), 2.34(\mathrm{~s}, 3 \mathrm{H})$, 2.79 (m, 3H), 3.17 (ddd, $J=3.7,12.2,13.4 \mathrm{~Hz}, 1 \mathrm{H}), 3.87$ (ddd, $J=3.7,10.4,13.4 \mathrm{~Hz}, 1 \mathrm{H}$ ), $4.26(\mathrm{dt}, J=13.2,4.8 \mathrm{~Hz}, 1 \mathrm{H}), 4.54(\mathrm{~m}, 1 \mathrm{H}), 4.86(\mathrm{~d}, J=12.0 \mathrm{~Hz}, 1 \mathrm{H}), 4.92(\mathrm{~d}, J=12.0 \mathrm{~Hz}$, 1H), 7.07-7.12(m, 4H), 7.27-7.30 (m, 3H), $7.53(\mathrm{~d}, J=8.4 \mathrm{~Hz}, 2 \mathrm{H}) .{ }^{13} \mathrm{C}$ NMR $(75.5 \mathrm{MHz}): \delta$ 21.0, 24.6, 29.8, 33.1, 39.8, 40.0, 67.8, 85.5, 128.0, 128.1, 128.2, 128.9, 135.3, 137.3, 138.2, 153.3, 167.3. MS (EI) m/z: $396\left(\mathrm{M}^{+}, 15\right), 351$ (4.8), 305 ( $\left.\mathrm{M}^{+}-\mathrm{Bn}, 6.8\right), 261$ (11), 187 (4.7); IR $v\left(\mathrm{~cm}^{-1}\right): 1703,1662$. Calcd for $\mathrm{C}_{22} \mathrm{H}_{24} \mathrm{~N}_{2} \mathrm{O}_{3} \mathrm{~S}: 396.1508$. Found: 396.1509 . 
( \pm -5-Benzyloxycarbonyl-6-(4-chlorophenyl)-1,5-diaza-7-thia-bicyclo[4.4.0]decan2-one (9d)<smiles>O=C(O)N1CCC(=O)N2CCCSC12[PH3+]</smiles>

Colorless oil; ${ }^{1} \mathrm{H}$ NMR (400 MHz): $\delta 1.71(\mathrm{~m}, 1 \mathrm{H}), 1.83(\mathrm{~m}, 1 \mathrm{H}), 2.20(\mathrm{~m}, 1 \mathrm{H}), 2.72(\mathrm{~m}, 1 \mathrm{H})$, $2.84(\mathrm{~m}, 2 \mathrm{H}), 3.14$ (ddd, $J=3.7,12.1,13.6 \mathrm{~Hz}, 1 \mathrm{H}), 3.87$ (ddd, $J=3.7,10.8,13.3 \mathrm{~Hz}, 1 \mathrm{H}$ ), $4.27(\mathrm{dt}, J=13.0,4.8 \mathrm{~Hz}, 1 \mathrm{H}), 4.53(\mathrm{~m}, 1 \mathrm{H}), 4.83(\mathrm{~d}, J=12.0 \mathrm{~Hz}, 1 \mathrm{H}), 4.91(\mathrm{~d}, J=12.0 \mathrm{~Hz}$, 1H), 7.07-7.09 (m, 2H), 7.21-7.32 (m, 5H), 7.55 (m, 2H). ${ }^{13} \mathrm{C}$ NMR (100 MHz): $\delta 24.4,29.8$, $33.1,39.9,40.0,68.1,85.1,128.35,128.40,128.42,129.65,129.74,134.3,135.0,139.1$, 153.3, 167.2. MS (EI) m/z: $416\left(\mathrm{M}^{+}, 12\right), 371$ (3.2), 281 (7.5), 211 (4.9); IR $v\left(\mathrm{~cm}^{-1}\right): 1700$, 1650. Calcd for $\mathrm{C}_{21} \mathrm{H}_{21} \mathrm{ClN}_{2} \mathrm{O}_{3} \mathrm{~S}$ : 416.0961. Found: 416.0962.

$( \pm)-3-B e n z y l o x y c a r b o n y l a m i n o m e t h y l t e t r a b e n z o[e, h, l, o]-7,14-\operatorname{dioxa}-1,10-\operatorname{diaza}-$ tricyclo $\left[9.5 .0 .0^{4,10}\right]$ hexadecan-2-one $(10 a)$<smiles>O=C1NCC2C(=O)N3c4ccccc4Oc4ccccc4C3N(c3ccccc3Oc3ccccc32)C2CCCCC12</smiles>

White solid; mp 250-251 ${ }^{\circ} \mathrm{C} ;{ }^{1} \mathrm{H}$ NMR (300 MHz): $\delta 3.22(\mathrm{~m}, 1 \mathrm{H}), 3.40$ (dt, $J=14.1,4.8 \mathrm{~Hz}$, $1 \mathrm{H}), 3.78(\mathrm{dt}, J=11.3,3.9 \mathrm{~Hz}, 1 \mathrm{H}), 4.60$ (d, $J=11.3 \mathrm{~Hz}, 1 \mathrm{H}), 4.76(\mathrm{~d}, J=12.5 \mathrm{~Hz}, 1 \mathrm{H}), 4.96$ $(\mathrm{d}, J=12.5 \mathrm{~Hz}, 1 \mathrm{H}), 5.38(\mathrm{t}, J=5.9 \mathrm{~Hz}, 1 \mathrm{H}), 6.44(\mathrm{~s}, 1 \mathrm{H}), 6.86-7.35(\mathrm{~m}, 20 \mathrm{H}), 7.55$ (d, $J=$ $7.8 \mathrm{~Hz}, 1 \mathrm{H}) .{ }^{13} \mathrm{C}$ NMR $(75.5 \mathrm{MHz}): \delta 39.6,48.3,56.5,66.4,78.1,119.7,120.8,121.6,121.9$, $122.6,124.4,125.3,125.8,126.1,126.9,127.5,127.7,127.8,128.2,128.4,129.0,129.6$, $130.1,130.6,131.8,132.8,136.5,139.2,148.3,153.8,156.4,156.6,158.6,170.4$. MS (EI) m/z: $595\left(\mathrm{M}^{+}, 1.0\right), 487$ (5.9), 431 (29), 309 (16), 292 (46), 195 (100); IR v (cm $\left.{ }^{-1}\right): 1716$, 1651. Calcd for $\mathrm{C}_{37} \mathrm{H}_{29} \mathrm{~N}_{3} \mathrm{O}_{5}$ : 595.2107. Found: 595.2097. 
( \pm )-3-Benzyloxycarbonylaminomethyl-2',2",-dimethyl-tetrabenzo[e,h,l,o]-7,14-dioxa $-1,10$-diaza-tricyclo[9.5.0.0 $\left.{ }^{4,10}\right]$ hexadecan-2-one (10b)<smiles>Cc1ccc2c(c1)C1C(CNC(=O)OCc3ccccc3)C(=O)N3c4ccccc4Oc4ccc(C)cc4C1N3c1ccccc1O2</smiles>

White solid; mp 135-137 ${ }^{\circ} \mathrm{C} ;{ }^{1} \mathrm{H}$ NMR (300 MHz): $\delta 2.29(\mathrm{~s}, 6 \mathrm{H}), 3.21(\mathrm{~m}, 1 \mathrm{H}), 3.40(\mathrm{~m}, 1 \mathrm{H})$, $3.80(\mathrm{dt}, J=11.4,4.5 \mathrm{~Hz}, 1 \mathrm{H}), 4.53(\mathrm{~d}, J=11.4 \mathrm{~Hz}, 1 \mathrm{H}), 4.84(\mathrm{~d}, J=12.6 \mathrm{~Hz}, 1 \mathrm{H}), 4.97(\mathrm{~d}, J$ $=12.6 \mathrm{~Hz}, 1 \mathrm{H}), 5.51(\mathrm{~s}, \mathrm{br}, 1 \mathrm{H}), 6.40(\mathrm{~s}, 1 \mathrm{H}), 6.85-7.32(\mathrm{~m}, 19 \mathrm{H}) .{ }^{13} \mathrm{C}$ NMR $(75.5 \mathrm{MHz}): \delta$ 20.7, 20.8, 39.7, 47.9, 56.7, 66.3, 78.1, 119.7, 120.5, 121.5, 121.7, 121.8, 122.6, 125.6, 126.0, $126.6,127.5,127.6,127.7,127.9,128.3,129.0,129.5,130.8,131.0,131.9,132.9,134.0$, 134.9, 136.7, 139.2, 148.5, 154.1, 154.6, 156.3, 156.5, 170.6. MS (EI) m/z: $623\left(\mathrm{M}^{+}, 0.3\right), 515$ (4.7), 459 (8.8), 306 (36), 250 (12); IR $v\left(\mathrm{~cm}^{-1}\right)$ : 1717, 1656. Calcd for $\mathrm{C}_{39} \mathrm{H}_{33} \mathrm{~N}_{3} \mathrm{O}_{5}: 623.2420$. Found: 623.2432 .

( \pm )-3-(3-Benzyloxycarbonylaminopropionyl)-2-benzylidene-1,3-thiazacyclohexane (11)<smiles>O=C(NCCC(=O)N1CCCSC1=Cc1ccccc1)c1ccccc1</smiles>

Colorless oil; ${ }^{1} \mathrm{H}$ NMR (300 MHz): $\delta 2.02$ (quintet, $\left.J=5.7 \mathrm{~Hz}, 2 \mathrm{H}\right), 2.73(\mathrm{t}, J=5.7 \mathrm{~Hz}, 2 \mathrm{H}$ ), $2.80(\mathrm{t}, J=5.7 \mathrm{~Hz}, 2 \mathrm{H}), 3.50(\mathrm{~m}, 2 \mathrm{H}), 3.85(\mathrm{~m}, 2 \mathrm{H}), 5.08(\mathrm{~s}, 2 \mathrm{H}), 5.57(\mathrm{~s}, \mathrm{br}, 1 \mathrm{H}), 6.61(\mathrm{~s}$, 1H), 7.25-7.47 (m, 10H). ${ }^{13} \mathrm{C}$ NMR (75.5 MHz): $\delta$ 24.6, 28.5, 33.8, 37.0, 44.4, 66.5, 127.9, 128.0, 128.3, 128.4, 129.1, 131.0, 133.4, 134.0, 136.6, 156.3, 171.4. MS (EI) m/z: $396\left(\mathrm{M}^{+}\right.$, 4.7), $305\left(\mathrm{M}^{+}-\mathrm{Bn}, 3.6\right), 288$ (17), 197 (22), 191 (100); IR $v\left(\mathrm{~cm}^{-1}\right)$ : 1717, 1652. Calcd for $\mathrm{C}_{22} \mathrm{H}_{24} \mathrm{~N}_{2} \mathrm{O}_{3} \mathrm{~S}: 396.1508$. Found: 396.1506 . 
$5 \mathbf{a}$<smiles>O=C(NCC1C(=O)[C@H](P)[C@H]1c1ccccc1)OCc1ccccc1</smiles>

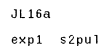

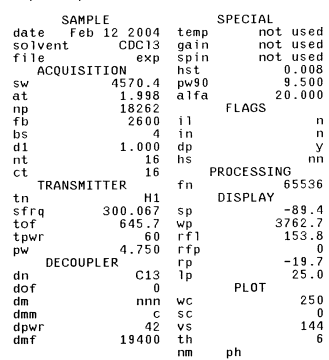

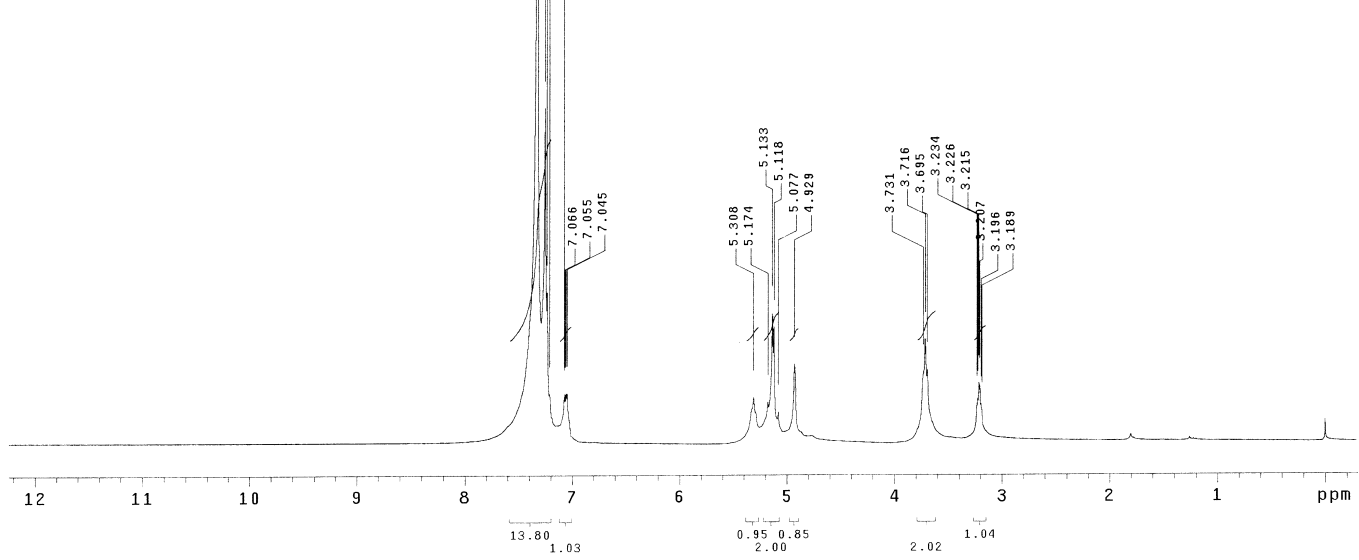

งเ16а
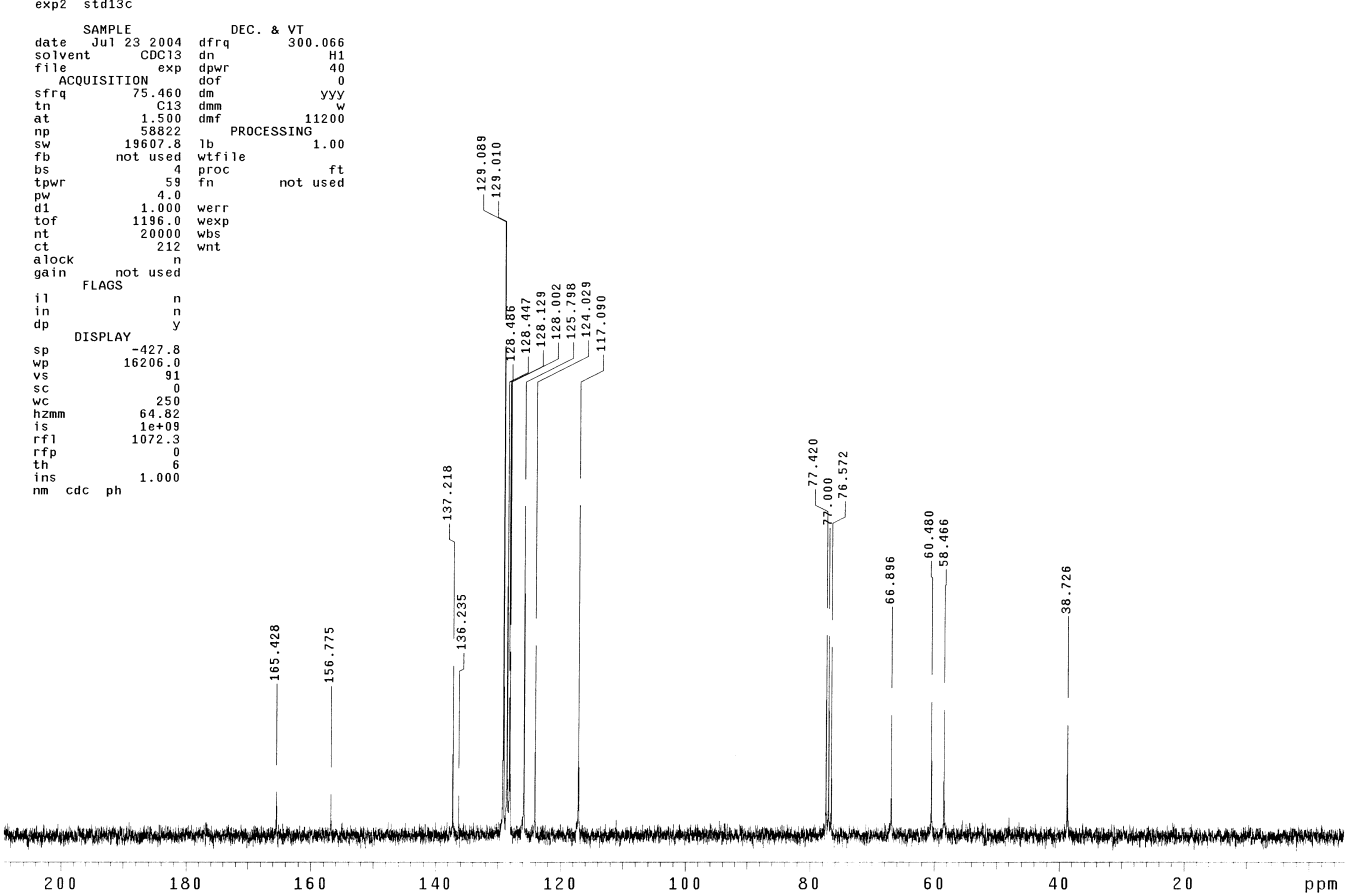
$5 b$<smiles>O=C(NC[C@@H]1C(=O)N(Cc2ccccc2)[C@H]1c1ccccc1)c1ccccc1</smiles>

JL137

$\begin{array}{lll}\text { exp2 } & \text { s2pu1 }\end{array}$

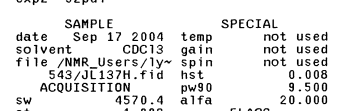

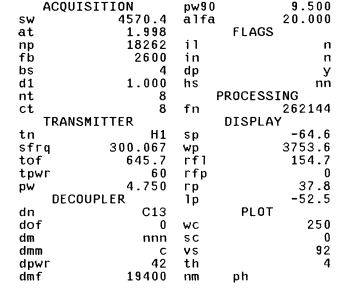

$( \pm)$

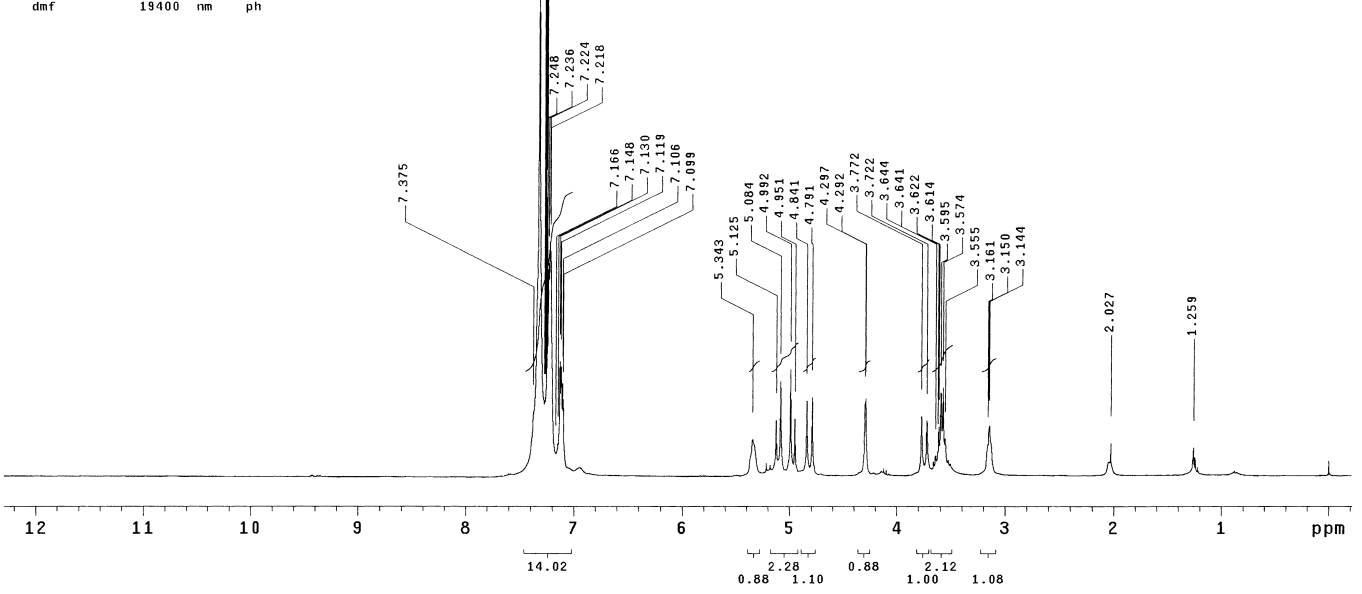

JL137
exp2 std13c
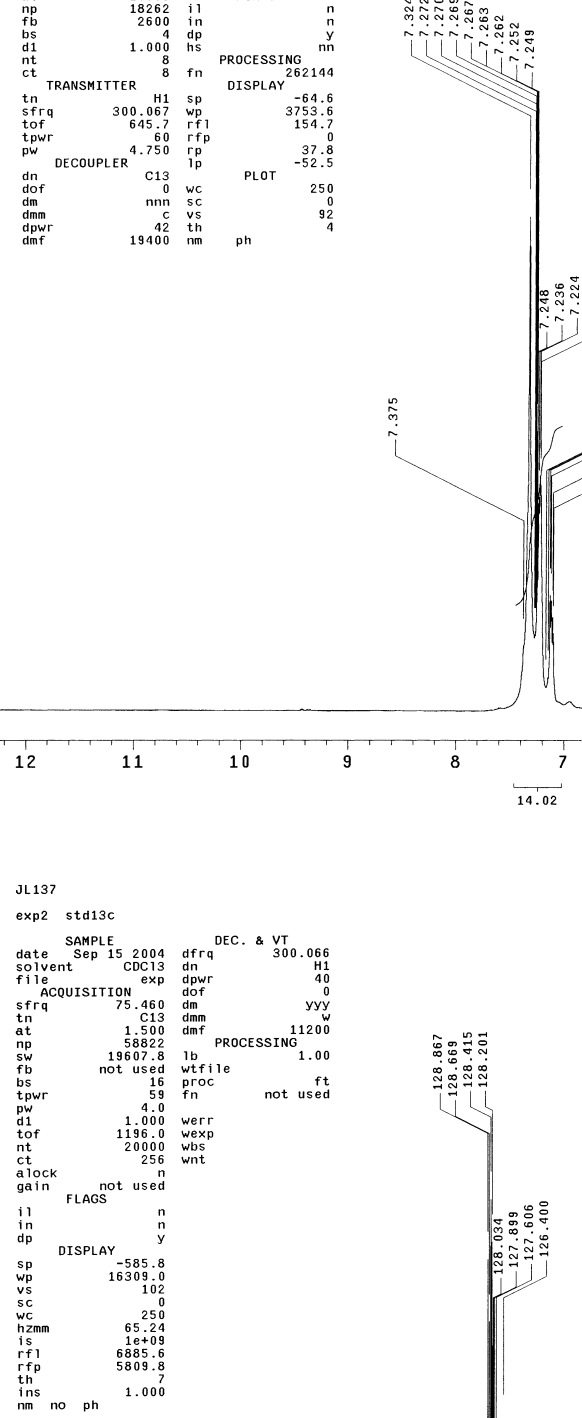
$5 c$<smiles>[13CH3]N1C(=O)C(CNC(=O)OCc2ccccc2)C1c1ccccc1</smiles>

JL140

exp1 s2pul

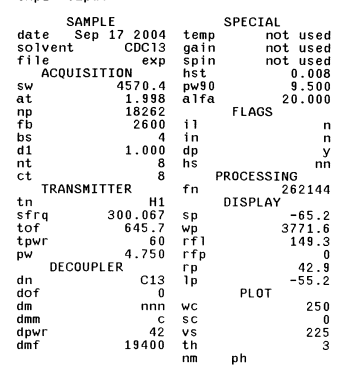

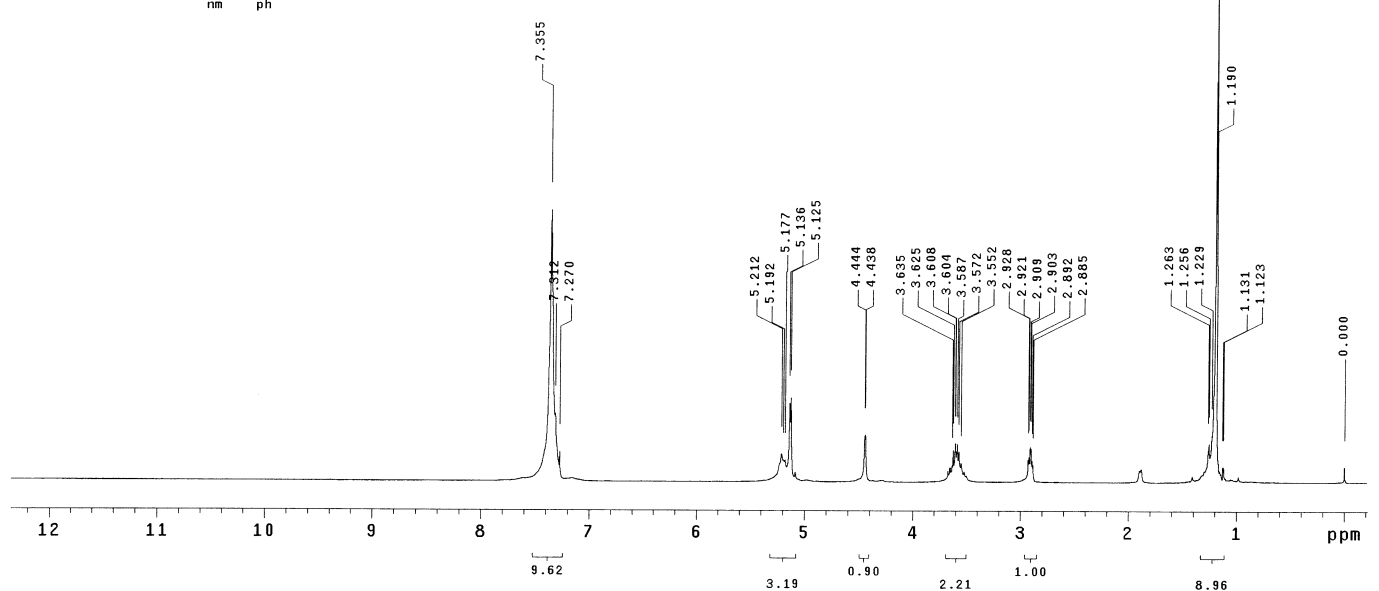

JL140

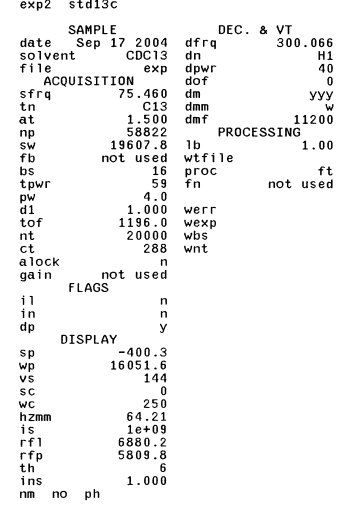


6a<smiles>O=C(NC[C@@H]1C(=O)N2CCCS[C@]12c1ccccc1)OCc1ccccc1</smiles>

$$
\begin{aligned}
& \text { JL25b-MW-ZN2+PhThiazine } \\
& \text { exp1 s2pul }
\end{aligned}
$$

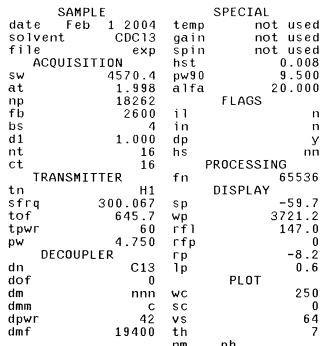

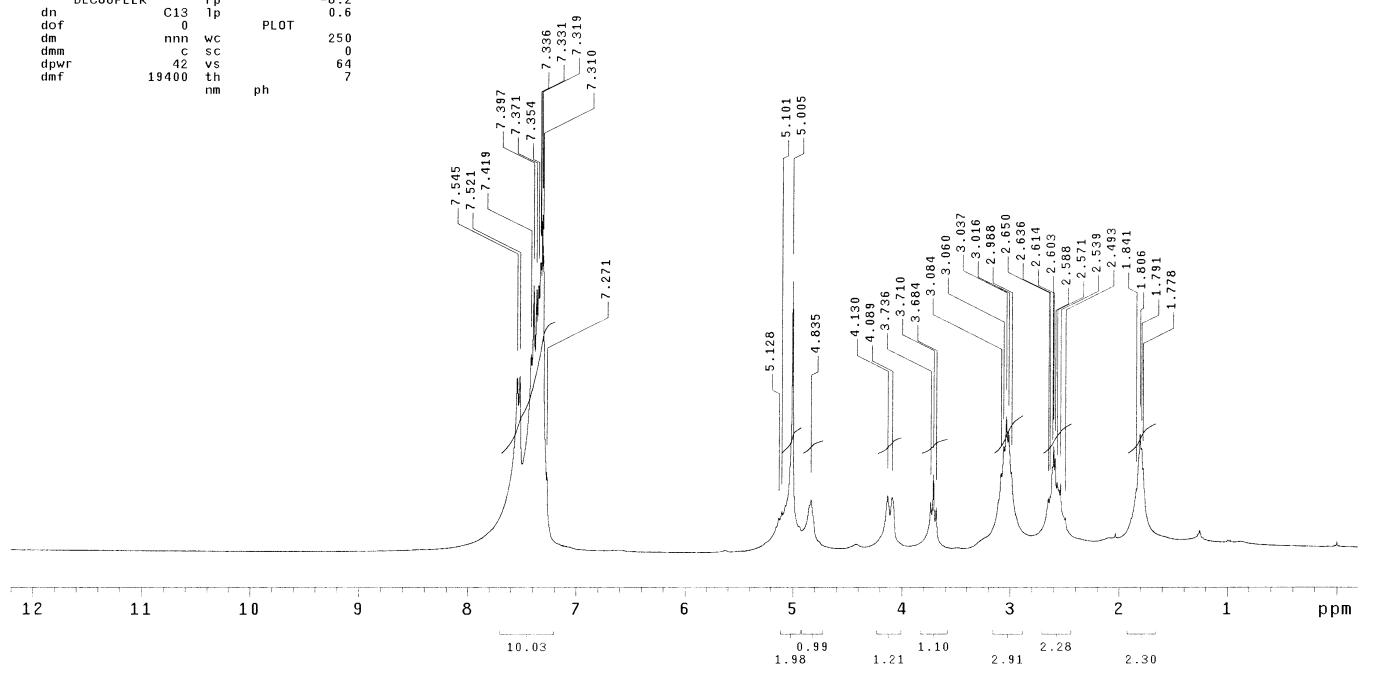

JL25b

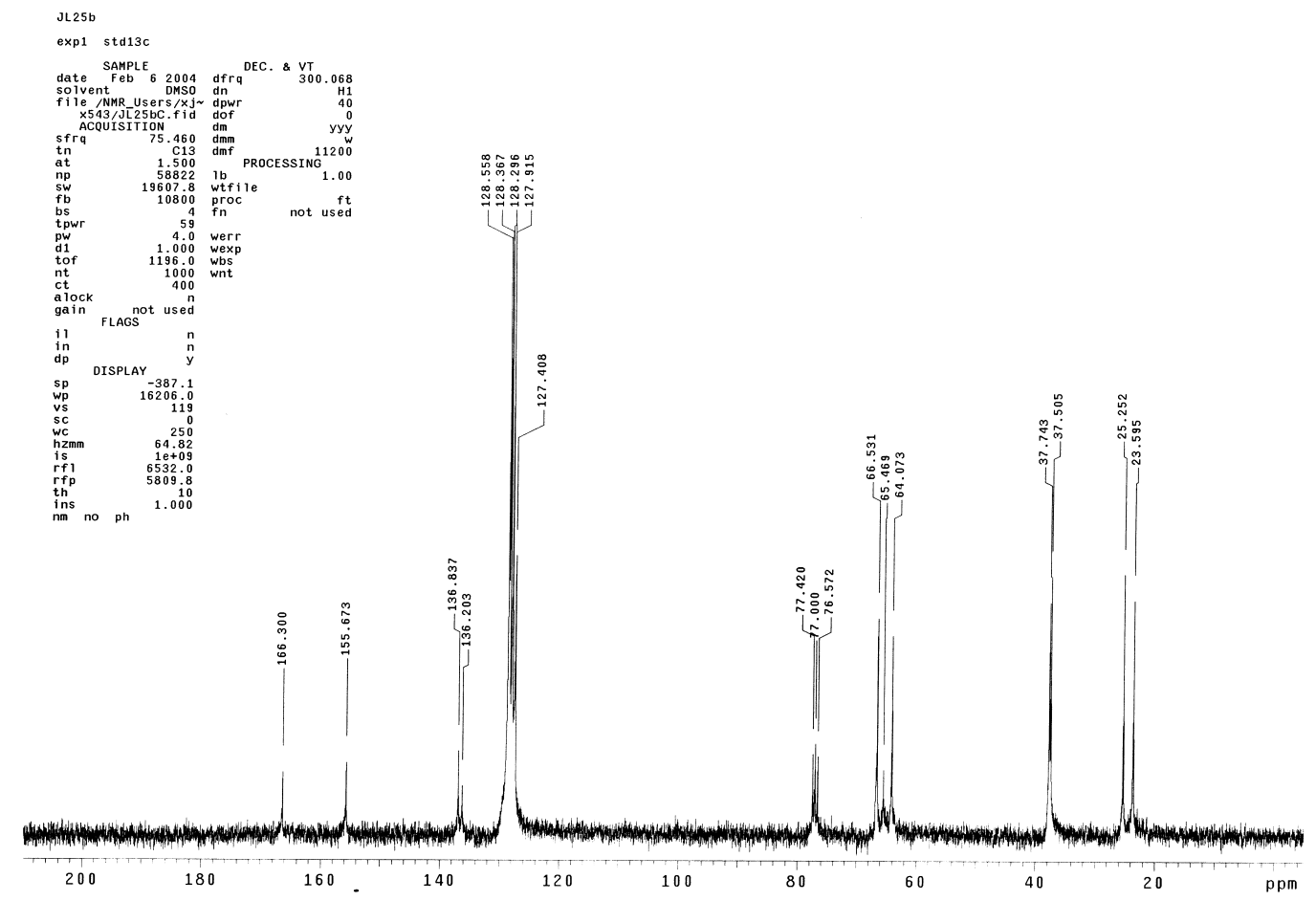


6b<smiles>CO[Pb]1(P)CCCN2C(=O)[C@H](CNC(=O)OCc3ccccc3)[C@]21P</smiles>

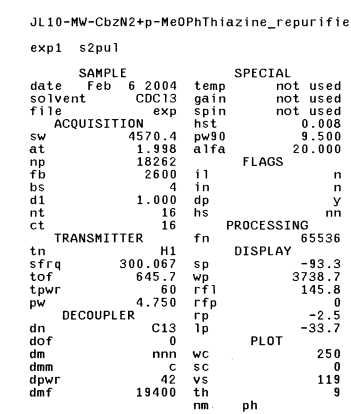

$( \pm)$

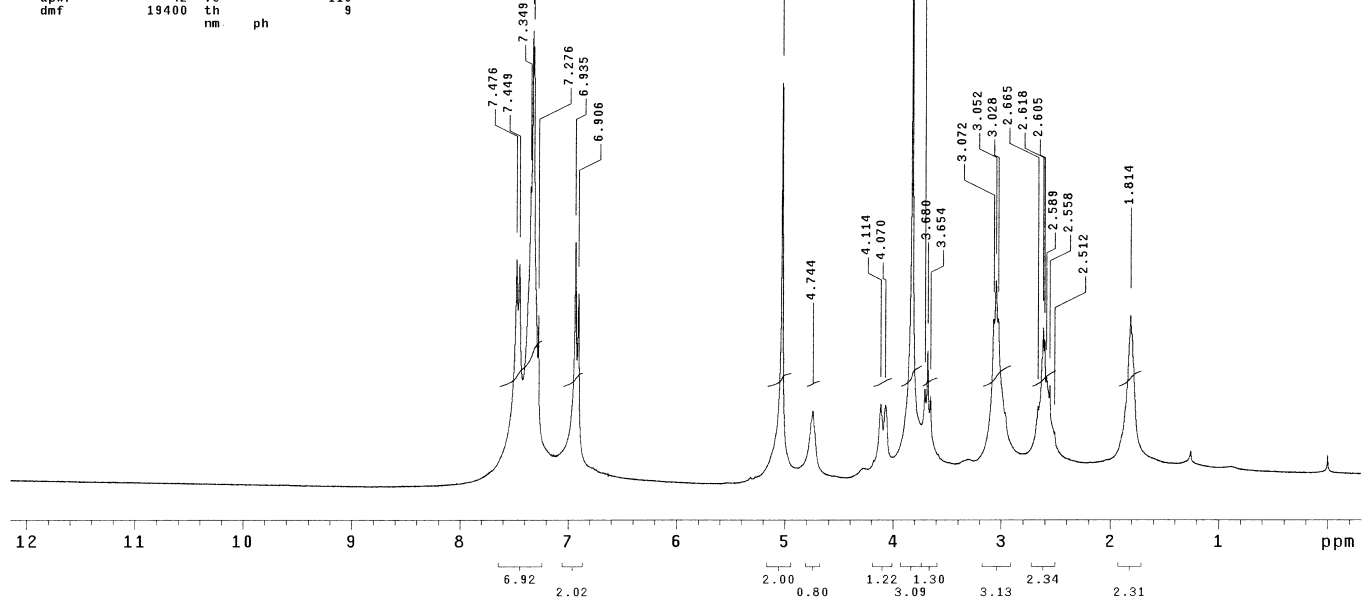

$J L 10-M w_{-} C$
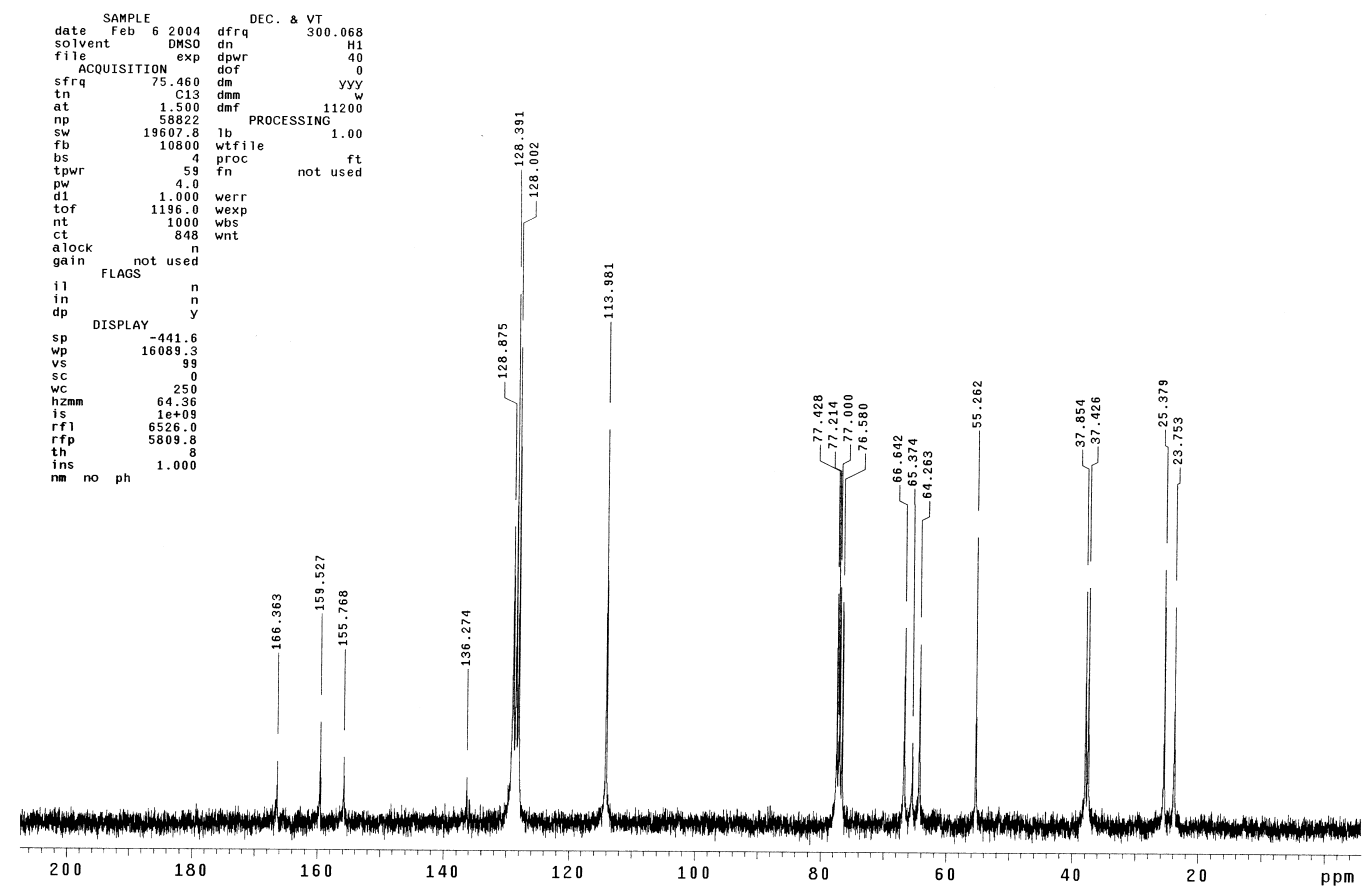
$6 c$

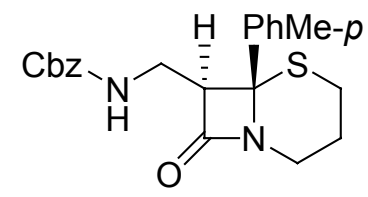
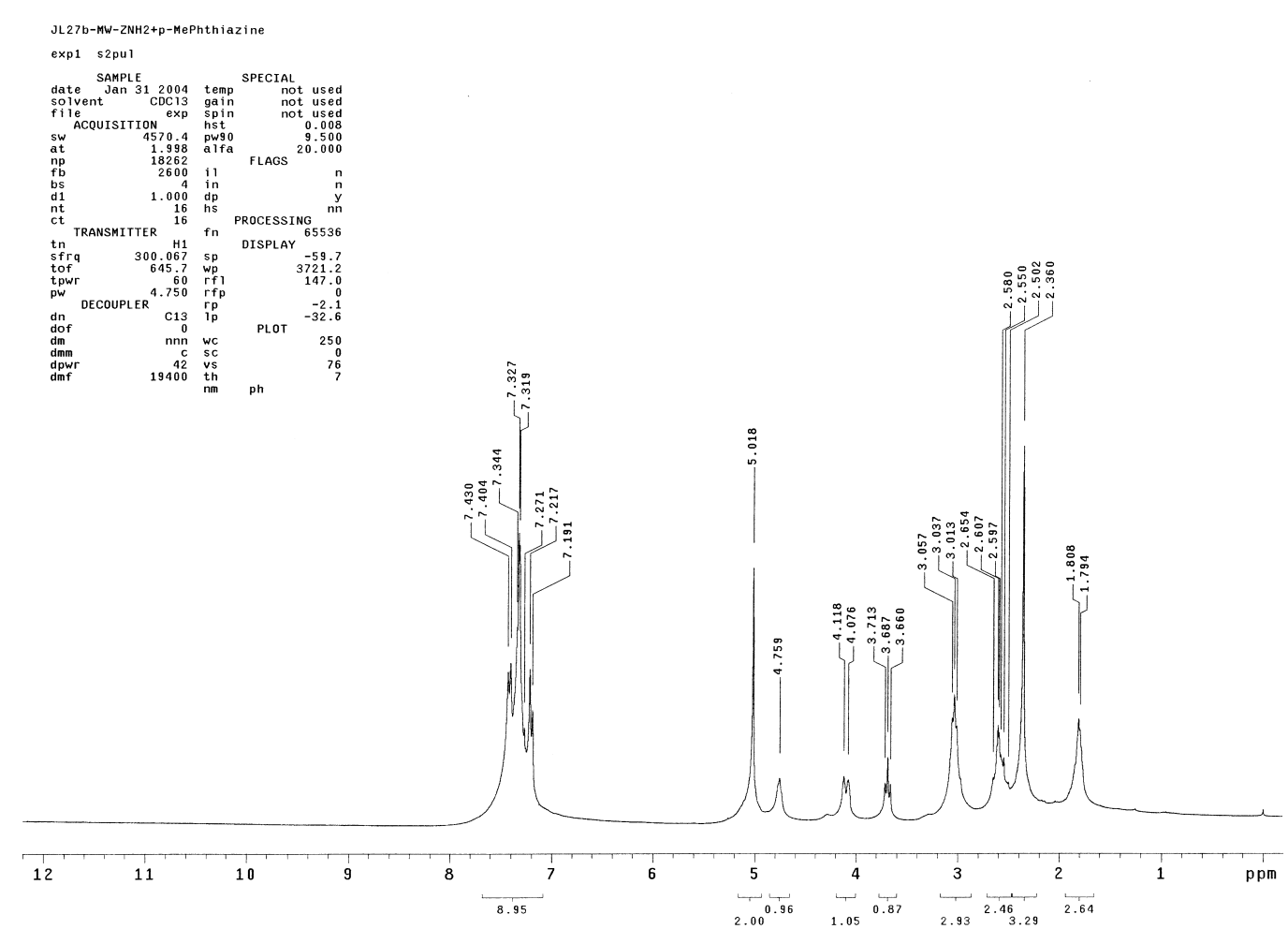

$\int_{\text {exp1 }}^{\pi \text { stat3c }}$

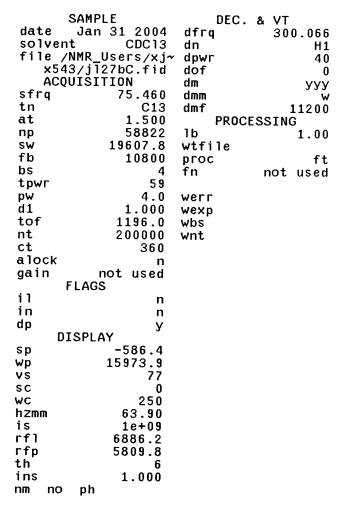


6d<smiles>O=C(NC[C@@H]1C(=O)N2CCCS[C@]12c1ccccc1)OCc1ccccc1</smiles>

$( \pm)$

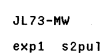

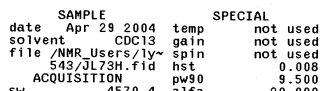

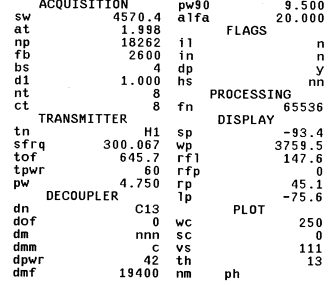

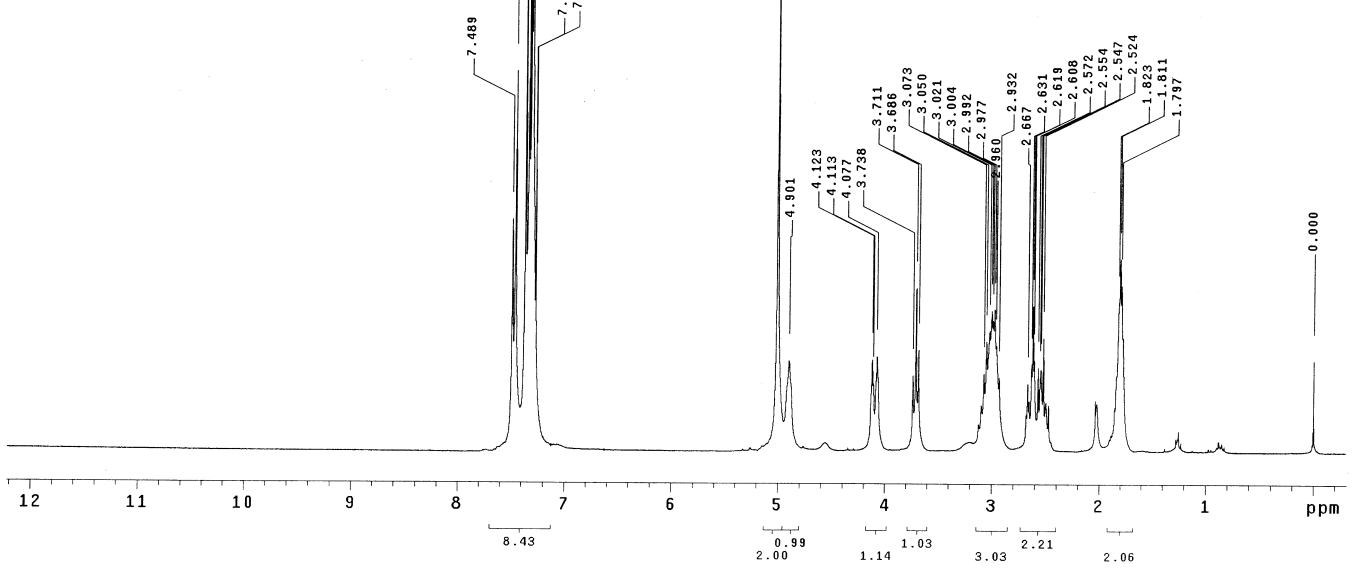

JL73-MW
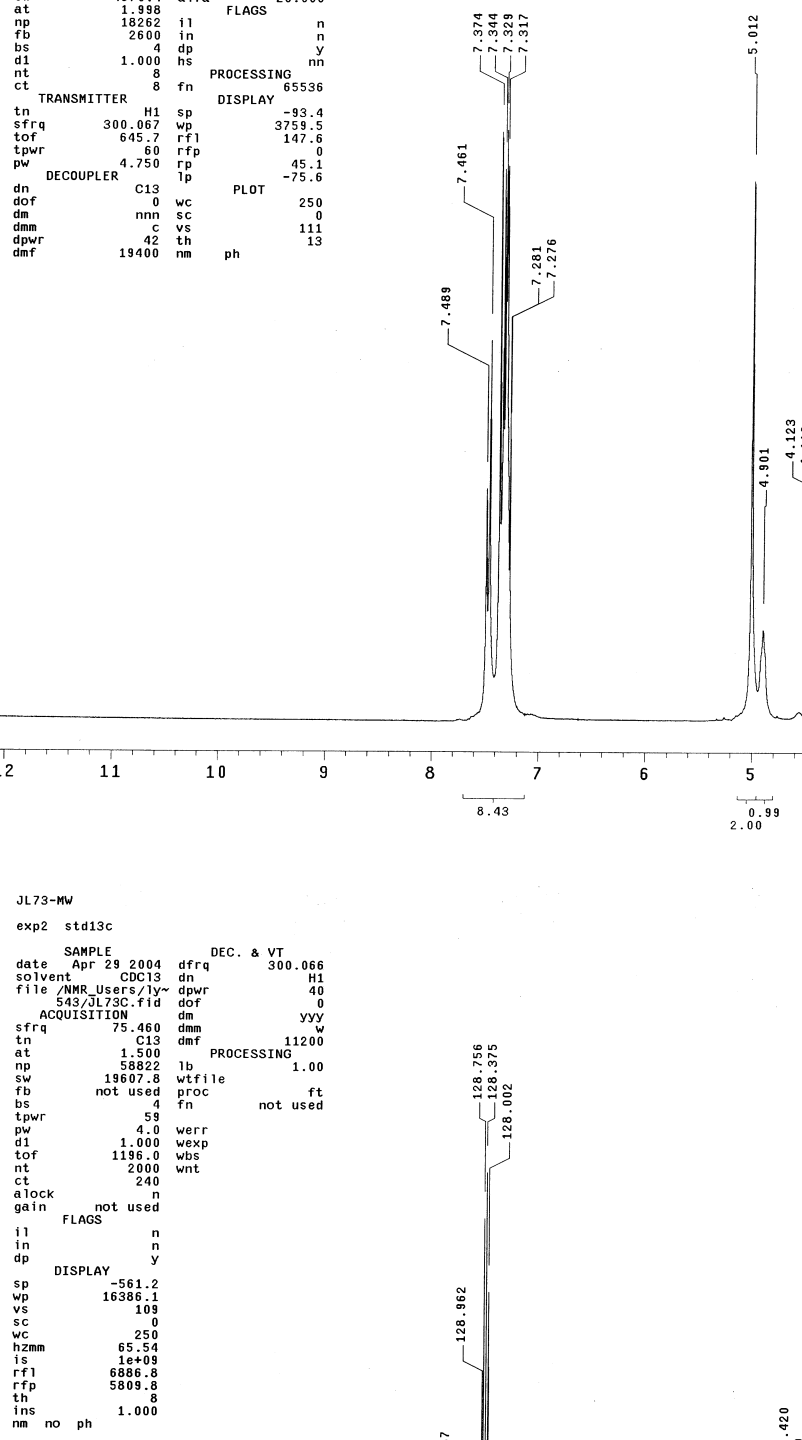
$7 \mathbf{a}$<smiles>O=C(NC[C@H]1C(=O)N2c3ccccc3Oc3ccccc3[C@H]12)OCc1ccccc1</smiles>

$( \pm)$

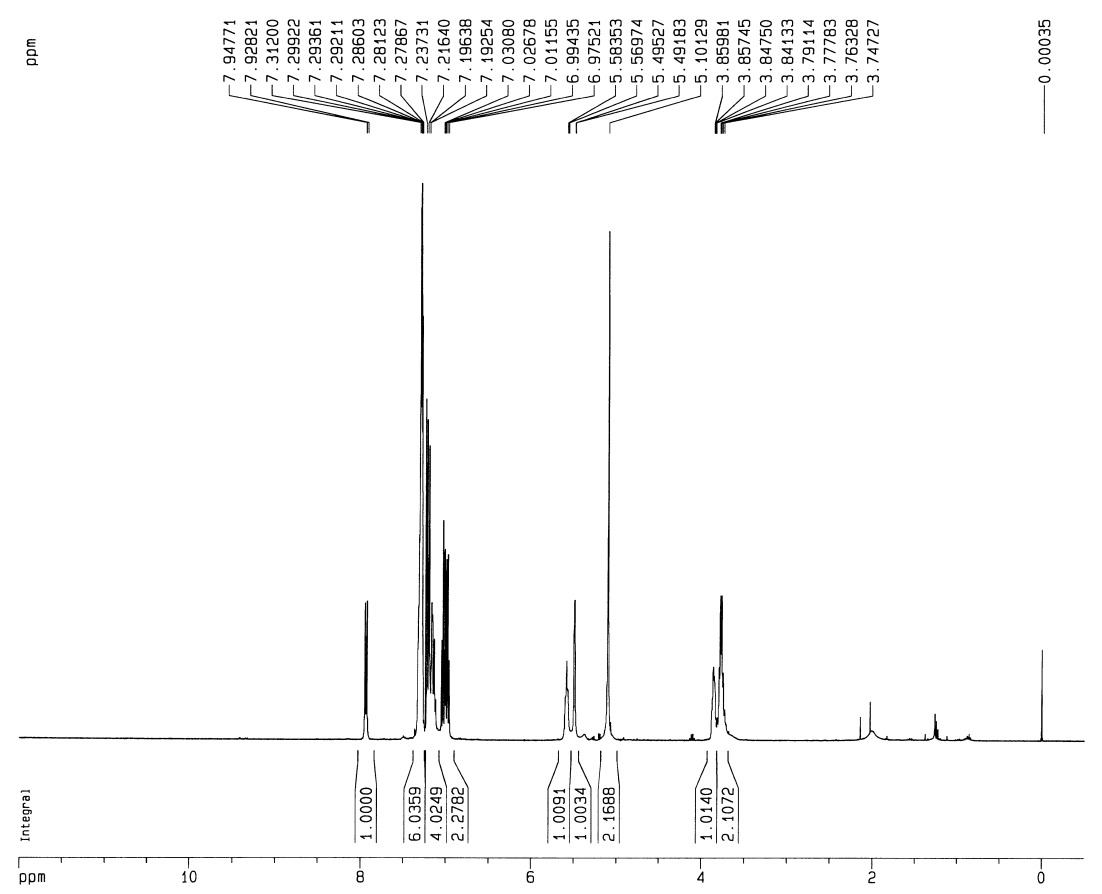

$\begin{array}{lr}\text { Current Data Parameters } \\ \text { NAME } & \text { H22441 } \\ \text { EXPNO } & 1 \\ \text { PROCNO } & 1\end{array}$

F2 - Acquisition Parameters

Date_ 20040727

$\begin{array}{ll}\text { Time } & 8.34 \\ \text { INSTRUM } & \end{array}$

ARXX00
INSTRUM 5 mm Multinu

PROBHD
PULPROG Multinu
2g

$\begin{array}{lr}\text { TD } & 32768 \\ \text { SOLVENT } & \text { CDC13 }\end{array}$

$\begin{array}{lr}N S & 54 \\ \text { DS } & 0\end{array}$

SWH $\quad 6410.256 \mathrm{~Hz}$

AQ $2.5559540 \mathrm{sec}$

$\begin{array}{lc}\mathrm{AQ} & 0.559540 \mathrm{sec} \\ \mathrm{RG} & 128\end{array}$

DW
DE
DE.

$\begin{array}{lr}\text { TE } & 300.0 \mathrm{~K} \\ \text { D } & 2.00000000 \mathrm{sec}\end{array}$

12.00 usec

$\begin{array}{lr}\text { DE } & 111.43 \mathrm{usec} \\ \text { SF01 } & 400.1320000 \mathrm{MHz}\end{array}$

NUCLEUS $1 \mathrm{H}$

F2 - Processing parameters

SI 32768

400.1300183 MHz

0
$-0.30 \mathrm{~Hz}$

$\mathrm{GB} \quad 0$

10 NMR plot parameters

$\begin{array}{ll}\text { CX } & 20.00 \mathrm{~cm}\end{array}$

$\begin{array}{lr}12.000 \mathrm{ppm} \\ \mathrm{F1} & 4801.56 \mathrm{~Hz}\end{array}$

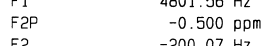

PPMCM $\quad-200.07 \mathrm{~Hz}$

HZCM $\quad 250.08125 \mathrm{~Hz} / \mathrm{cm}$

JL117re

$\exp 2 \quad \operatorname{std13c}$

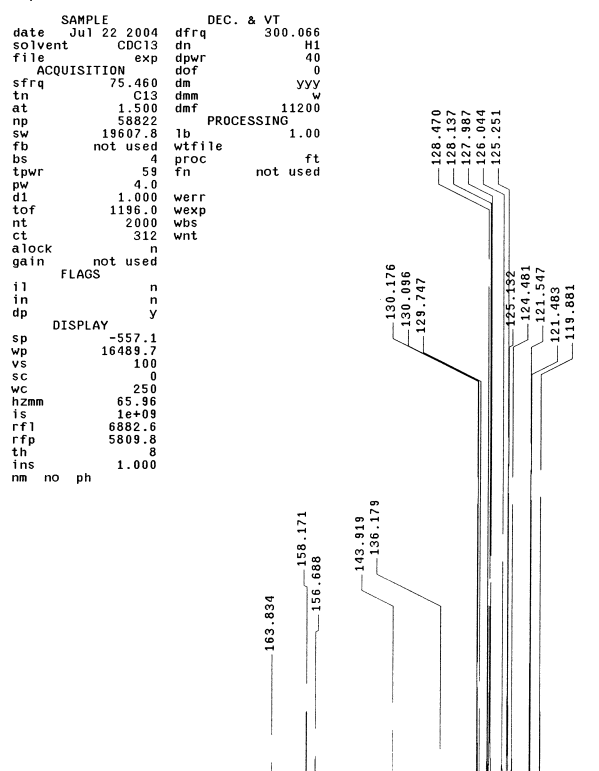

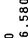

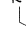

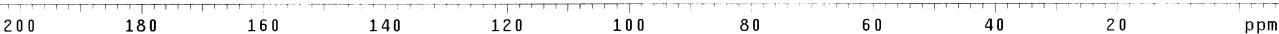




\section{DEPT of $7 \mathbf{a}$}

งเ115-3

Pulse Sequence: dept

CH3 carbons

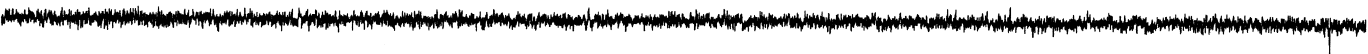

CH2 Carbons
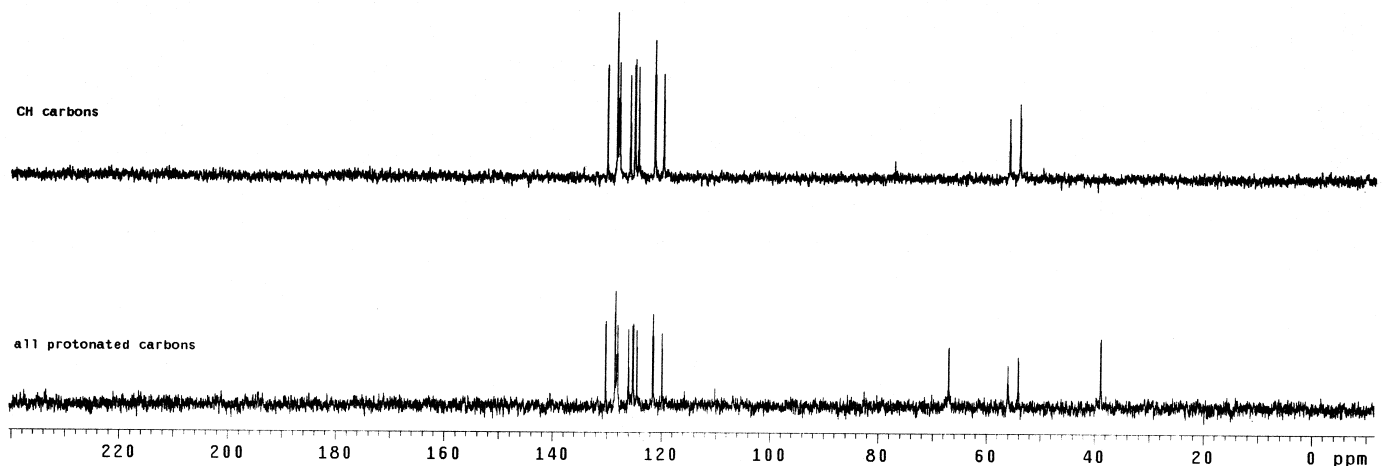

\section{gHSQC of 7a}

JL115-3

Archlve directory: /export/home/vnmr 1/vnmrsys/data
Sample directory:

Sample directory
File: PROTON

Pulse Sequence: gHSOC

Solvent: CDC13 $_{\text {Ambient temperature }}$
Mercury-3000B "

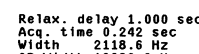

20 width $1386.9 \mathrm{H}$
4 repet
2

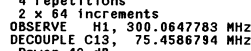

Power 42 dB'
on dur ing acquisition
off during deeay

Off dur ing de lay
GARP - 1 modulated
DATA PROCESST

DATA PROCESSING
Gauss apodization $0.111 \mathrm{sec}$
F1

F1 DATA PRRCESSING

FT size $1024 \times 2048 \times 28 \mathrm{sec}$
Total time $12 \mathrm{~min}, 52 \mathrm{sec}$

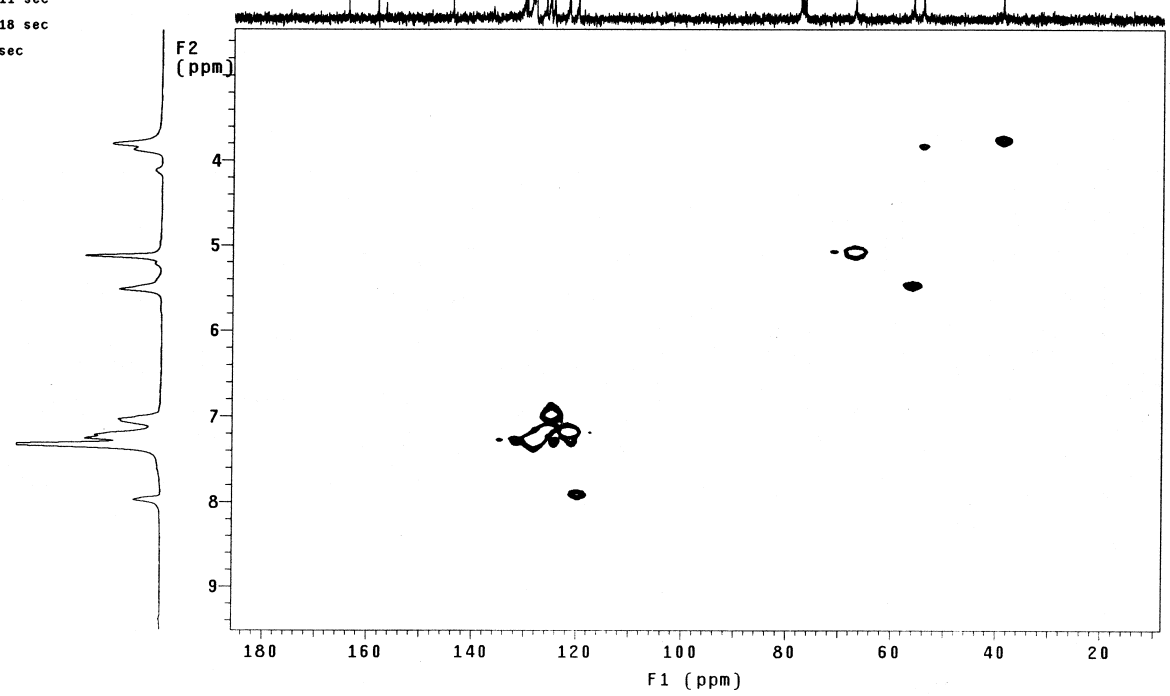


$7 b$<smiles>Cc1ccc2c(c1)[C@H]1C[C@H](CNC(=O)OCc3ccccc3)C(=O)N1c1ccccc1O2</smiles>

$( \pm)$
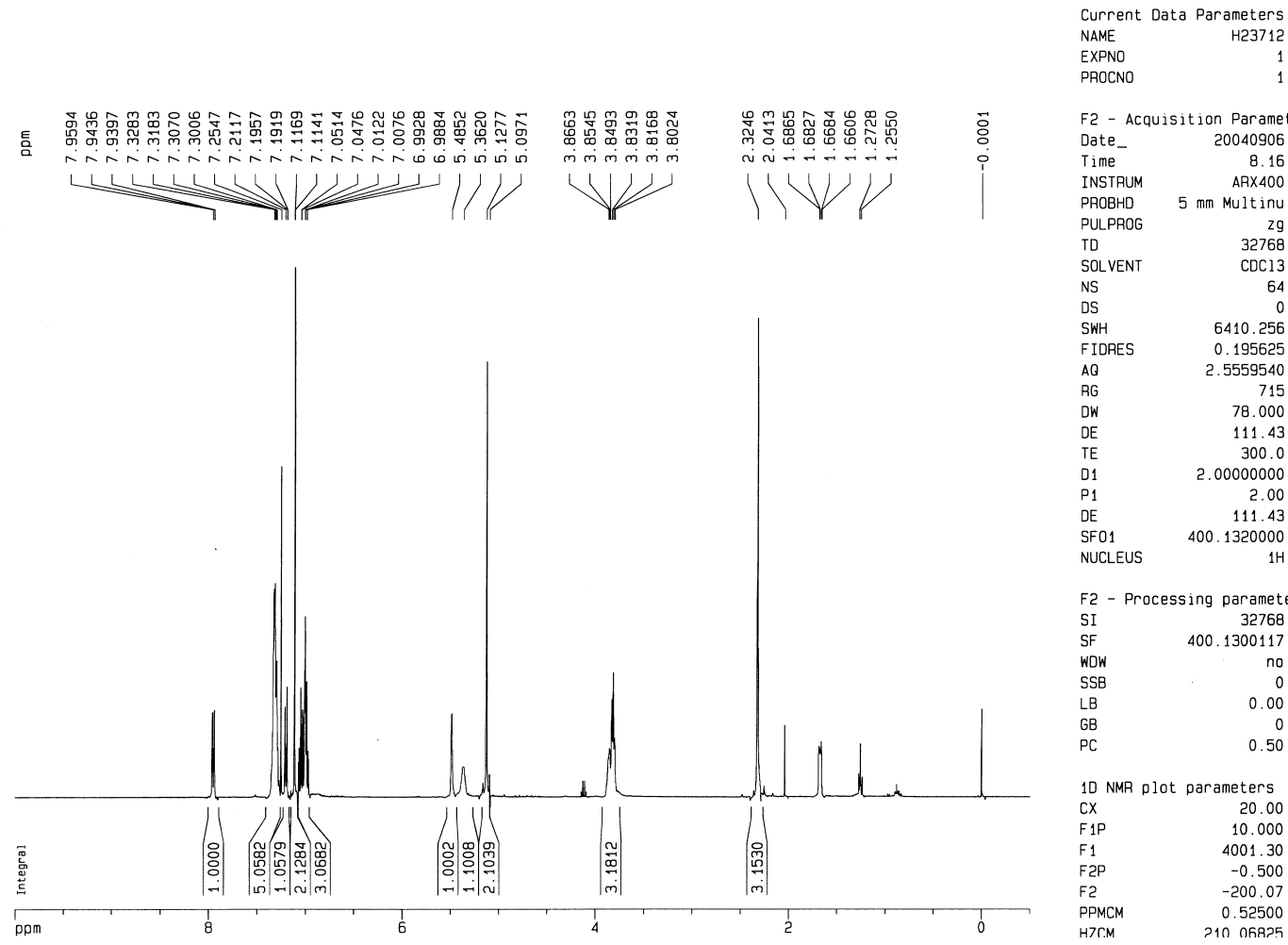

F2 - Acquisition Parameters

Date_ 20040906

$\begin{array}{ll}\text { Time } & 8.16\end{array}$

INSTRUM ARX400

PROBHD $5 \mathrm{~mm}$ Multinu

$\begin{array}{lr}\text { PULPROG } & 29 \\ \text { TD } & 32768\end{array}$

$\begin{array}{lr}\text { SOLVENT } & \text { COC13 } \\ \text { NS } & 64\end{array}$

$\begin{array}{lr}\text { DS } & 0 \\ \text { SWH } & 6410.256 \mathrm{~Hz}\end{array}$

FIDRES $\quad 0.195625 \mathrm{~Hz}$

AQ $\quad 2.5559540 \mathrm{se}$

DW $\quad 78.000$ use

111.43 usec

$2.00000000 \mathrm{sec}$

$\begin{array}{lr}\text { P1 } & 2.00000 \mathrm{usec} \\ \text { DE } & 111.43 \mathrm{usec} \\ \text { SF01 } & 400.1320000 \mathrm{MHz}\end{array}$

NUCLEUS 400.13200

F2 - Processing parameters

$\begin{array}{lc}\text { SI } & 32768 \\ \text { SF } & 400.1300117 \mathrm{MHz}\end{array}$

WOW
SSB

$\begin{array}{lc}\text { LB } & 0.00 \mathrm{~Hz} \\ \text { GB } & 0 \\ \text { PC } & 0.50\end{array}$

10 NMR plot parameters

$20.00 \mathrm{~cm}$

$\begin{array}{ll}\text { F1P } & 10.000 \mathrm{ppm}\end{array}$

$\begin{array}{ll}\text { F1 } & 4001.30 \mathrm{~Hz} \\ \text { F2P } & -0.500 \mathrm{ppm}\end{array}$

$\begin{array}{ll}\text { F2P } & -0.500 \mathrm{ppm} \\ \text { F2 } & -200.07 \mathrm{~Hz}\end{array}$

$\begin{array}{ll}\text { F2 } & -200.07 \mathrm{~Hz} \\ \text { PPMCM } & 0.52500 \mathrm{ppm} / \mathrm{cm}\end{array}$

JL133

$0.52500 \mathrm{ppm} / \mathrm{cm}$

exp2 std13c
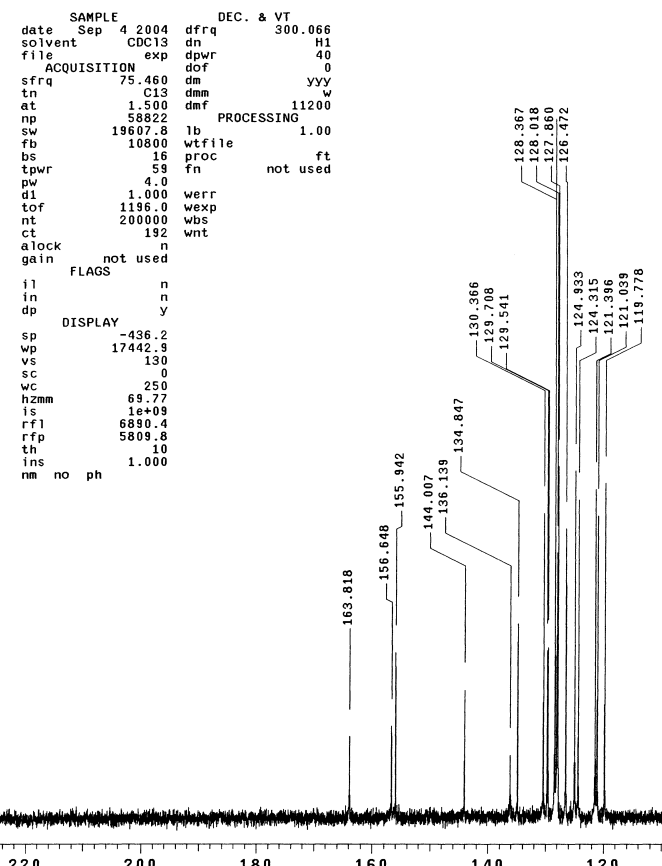
<smiles>O=C(OCc1ccccc1)N1CCC(=O)N(c2ccccc2)C1c1ccccc1</smiles>

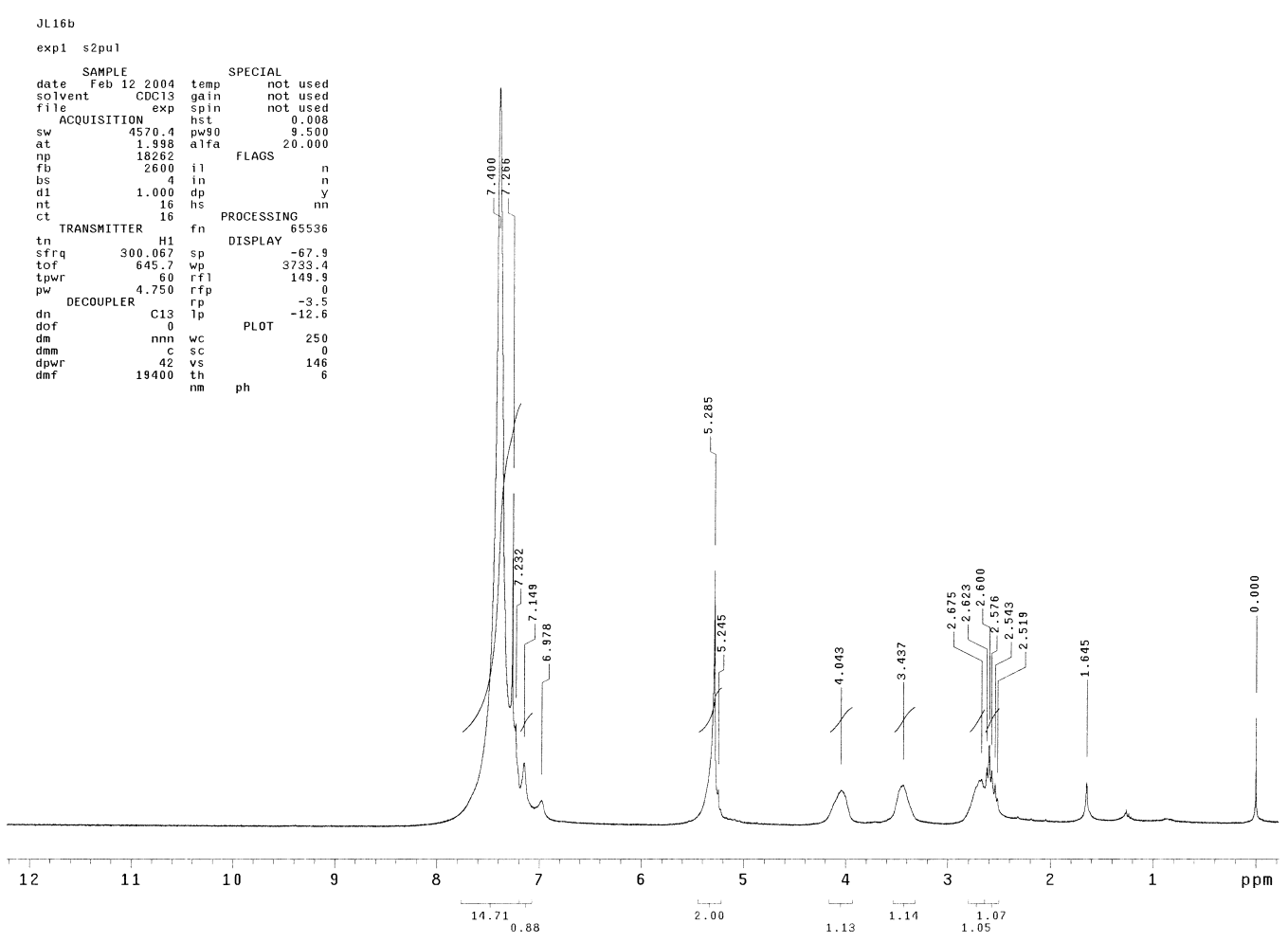

JL16bre
exp2 2 std13
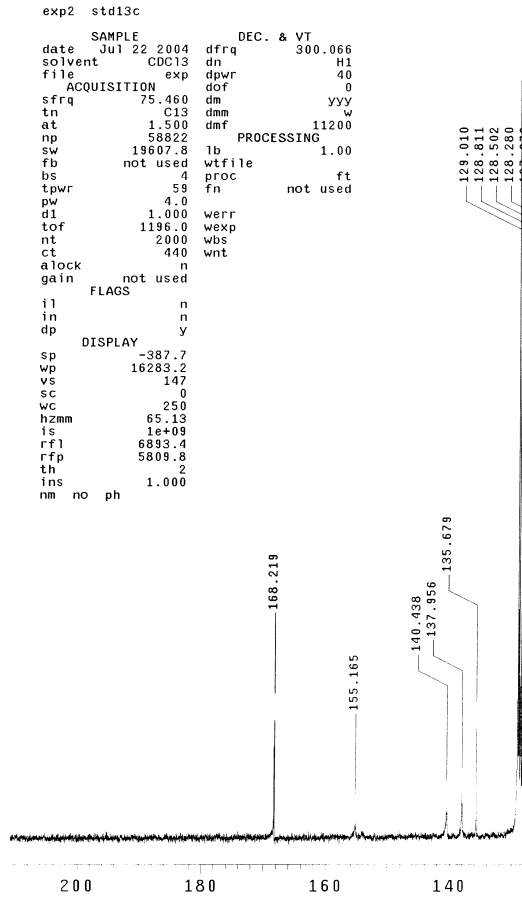
9a<smiles>O=C1CCN(C(=O)OCc2ccccc2)C2(P)SCCCN12</smiles>

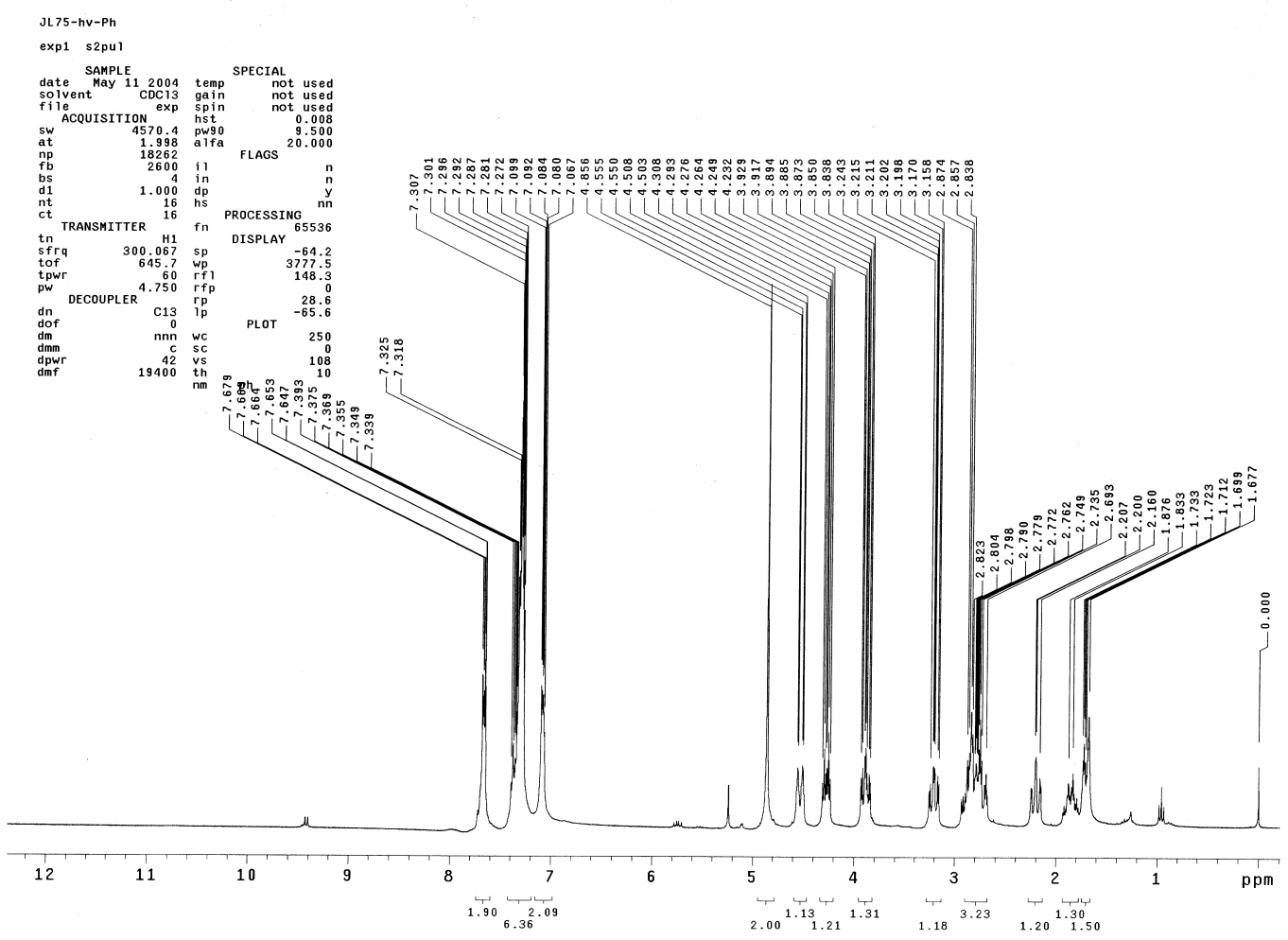

JL75-hv-ph
exp2 std13c
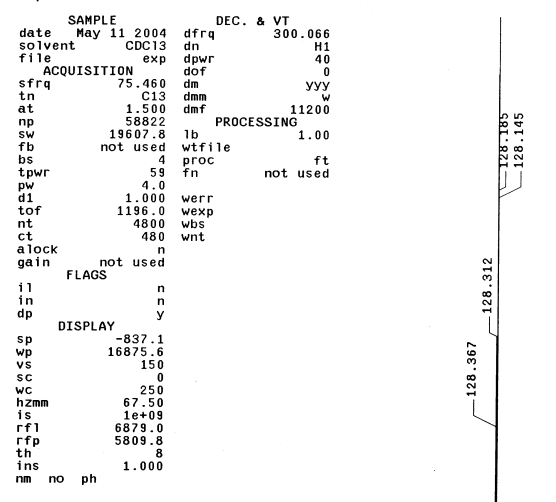

$200 \quad 180$

$160 \quad 140$

140

120

100
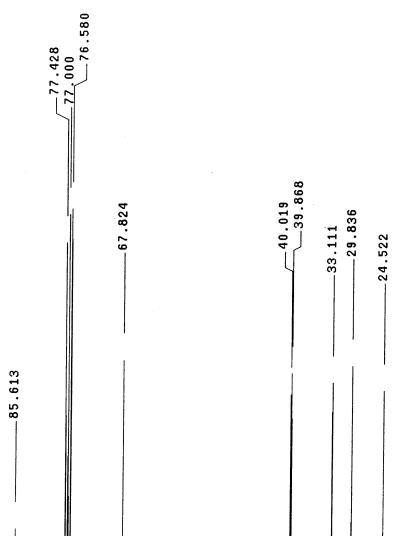


\section{DEPT of 9a}

JL75-hv

Pulse Sequence: dept

СH3 carbons

CH2 carbons
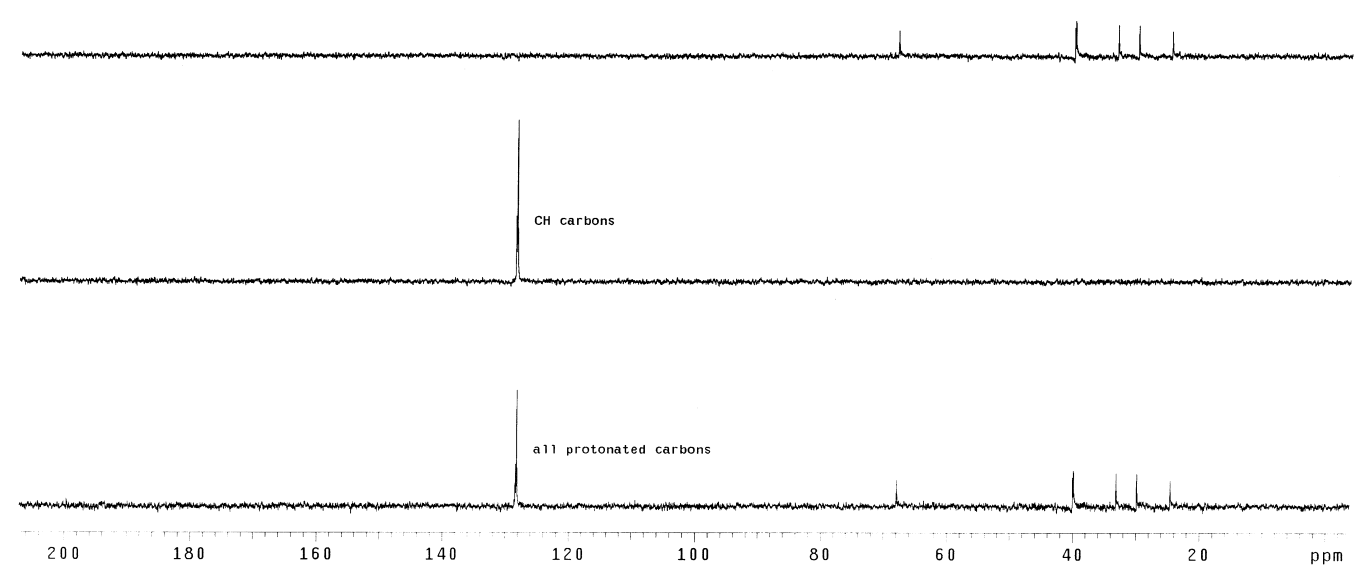

\section{gHSQC of 9a}

JL75-hv-Ph

Archive directory: /export/home/vnmr1/vnmrsys/data
sample directory:

Pulse Sequence: gHsec

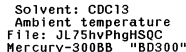

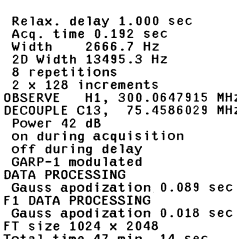

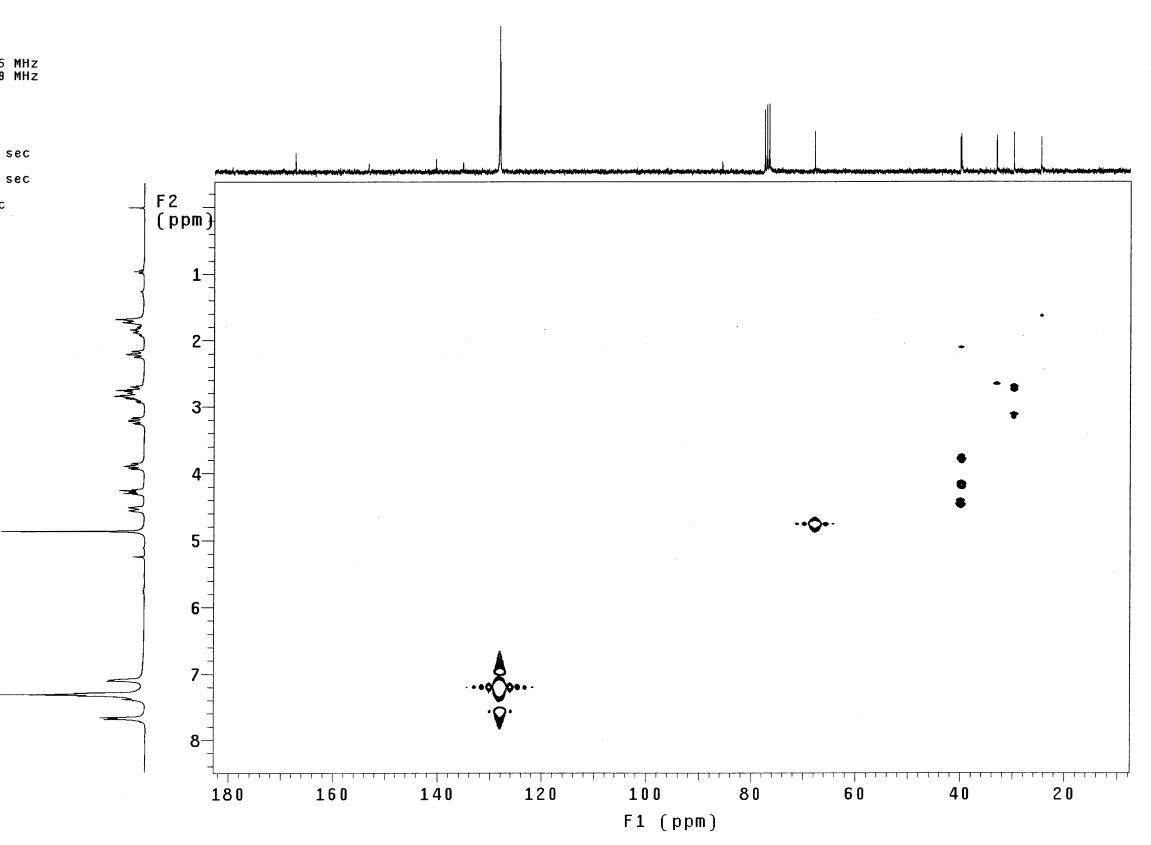




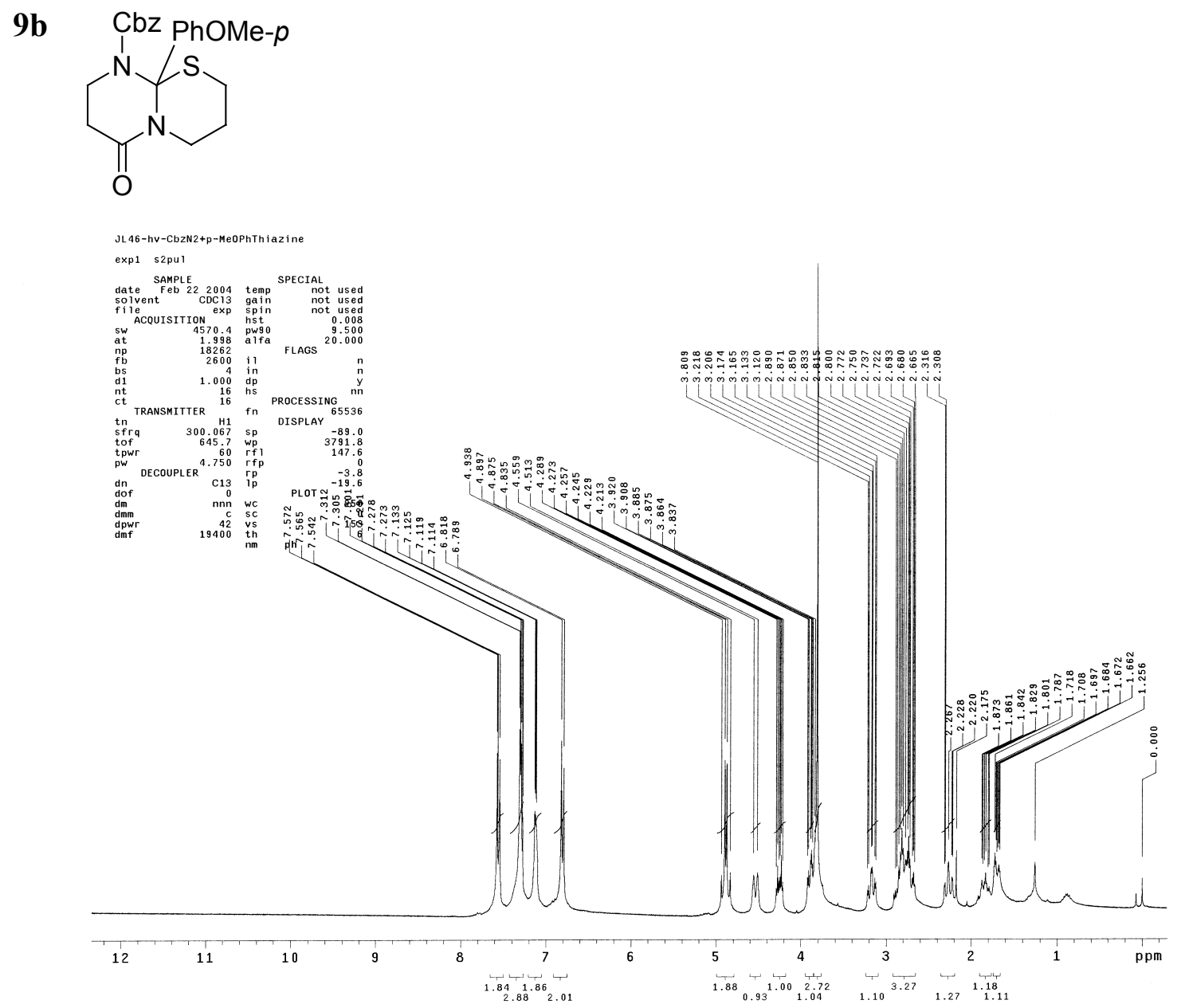

JL46-hv
exp2 $\quad$ std13
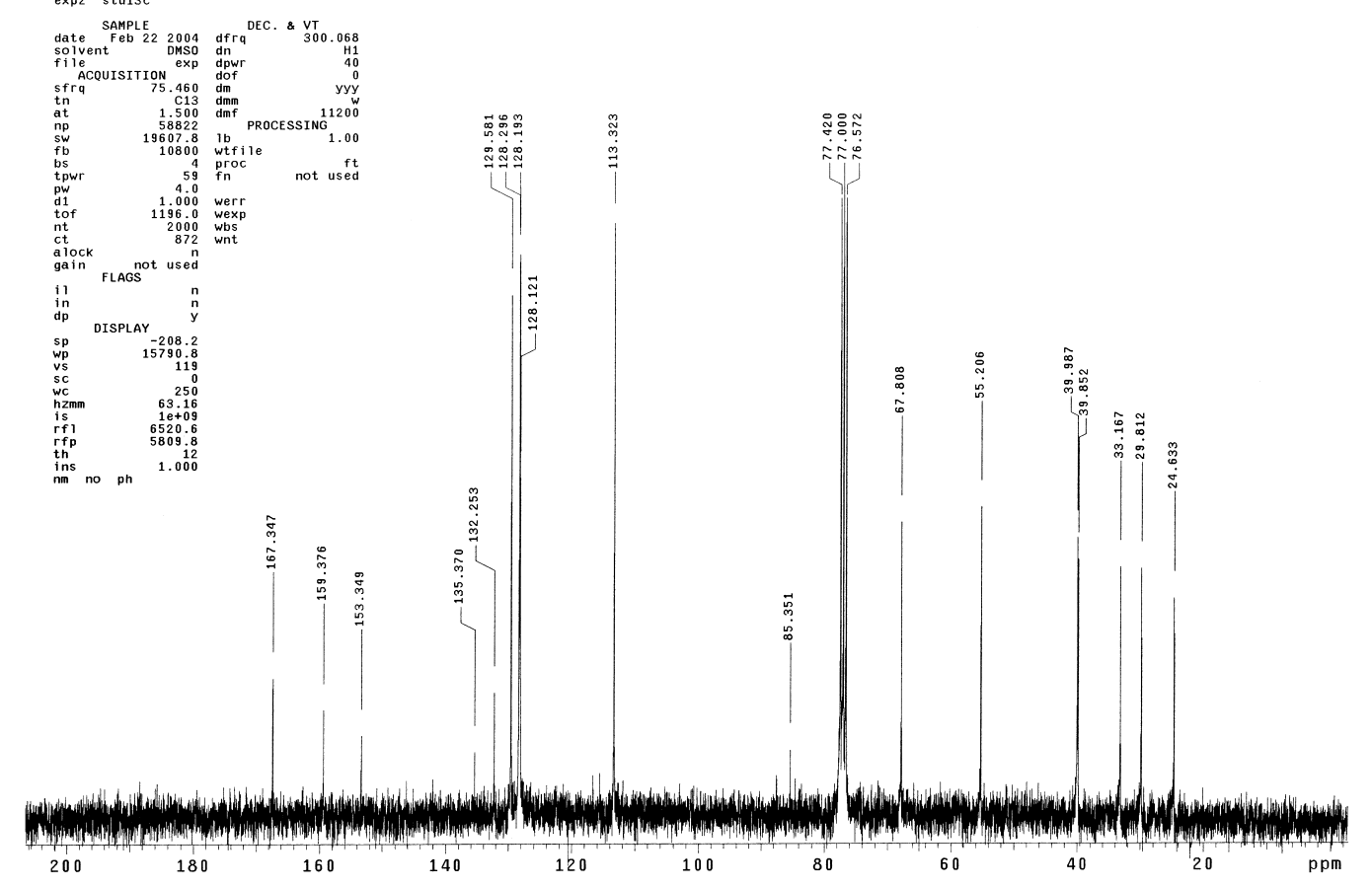
9c<smiles>CC(=O)N1CCC(=O)N2CCCSC12P(C)(=O)c1ccccc1</smiles>

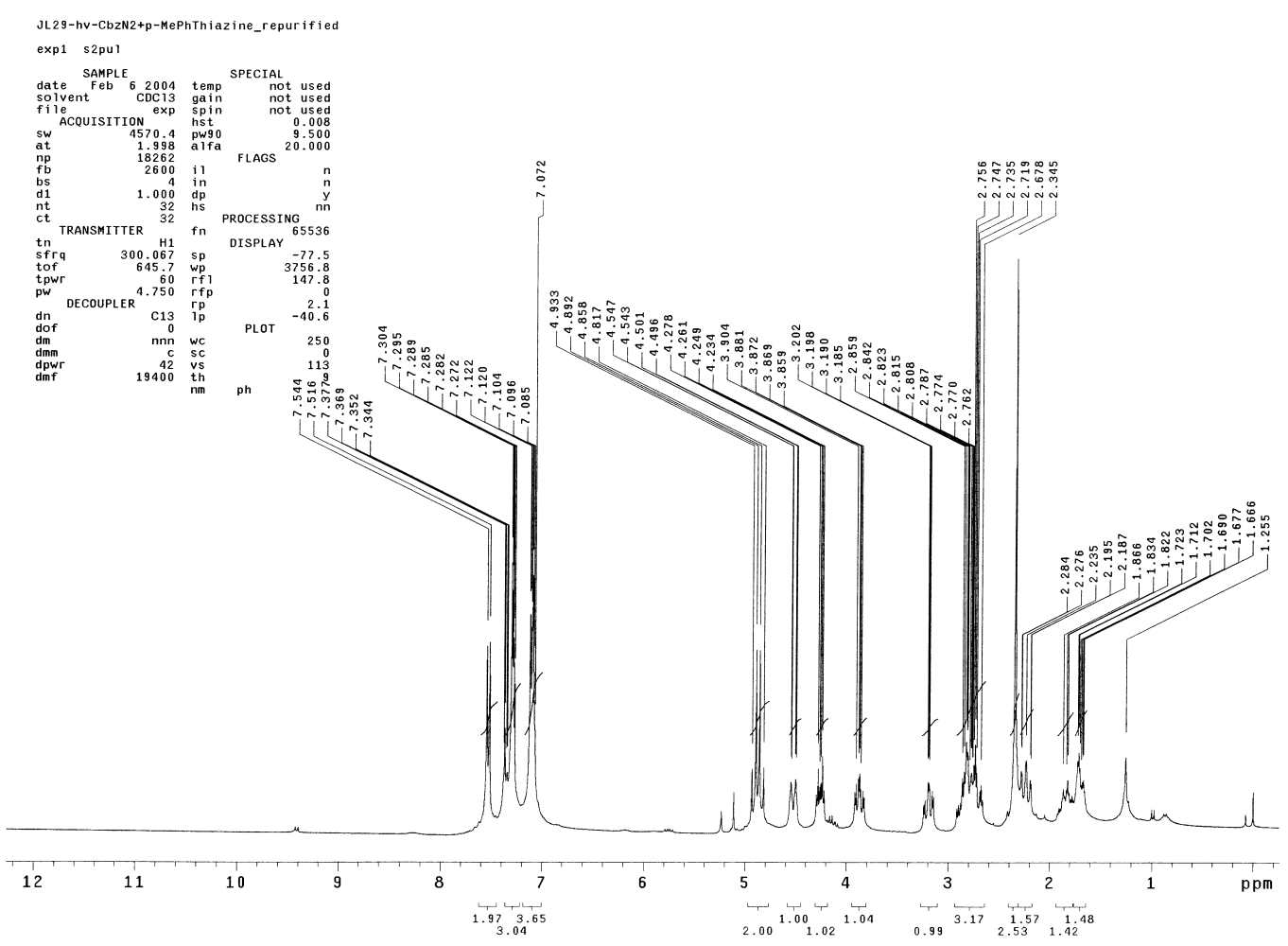

JL29-hv-C_repurified

exp2 std13c

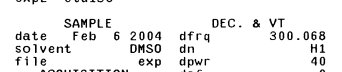

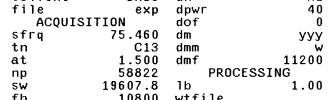

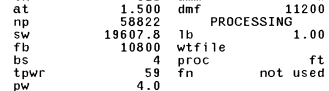

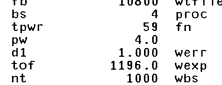

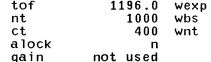

i1 FLAGS ${ }^{\text {not }}$ use

$\begin{array}{ll}\text { dp } & \text { DISPLAY } \\ \text { sp } \\ \text { wp } & 16160 .\end{array}$

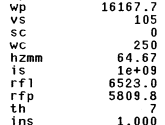

(n)

-

ins no ph 1.000 
9d<smiles>O=C(O)PC12SCCCN1C(=O)CCN2C(=O)O</smiles>

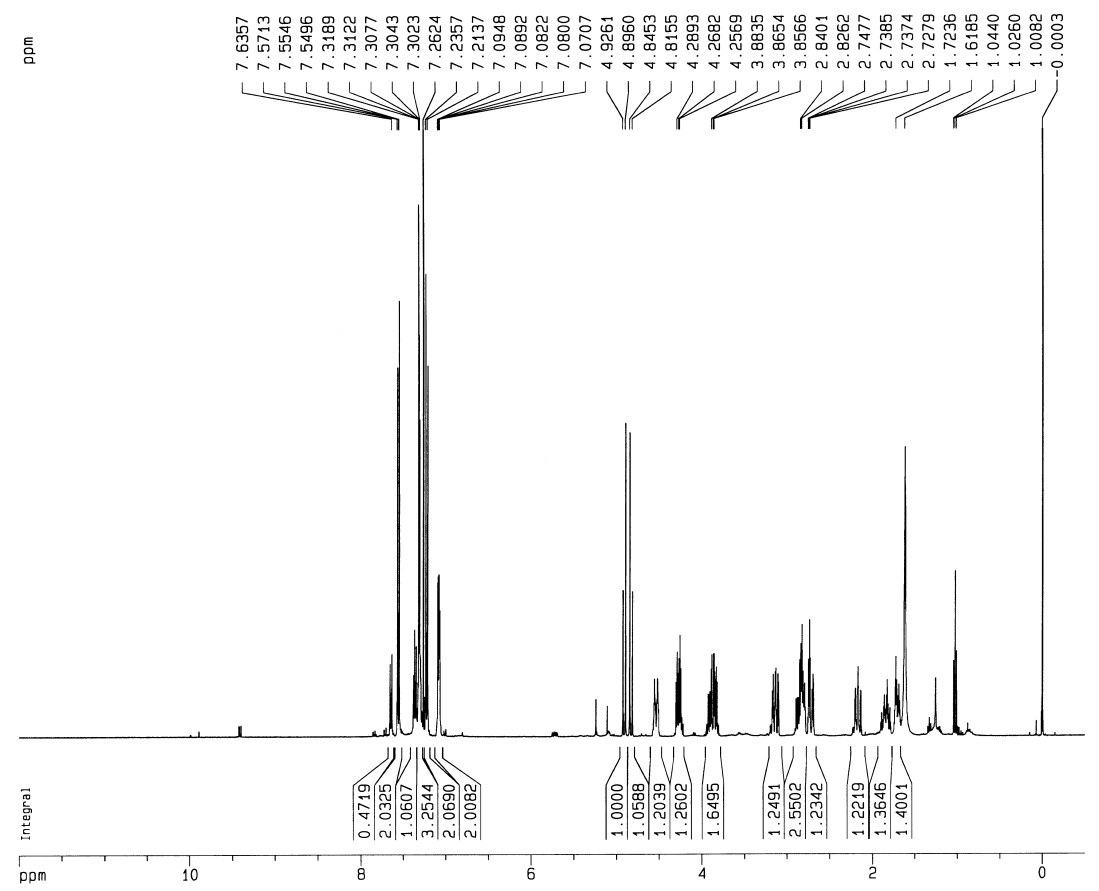

$\begin{array}{lr}\text { Current } & \text { Data Parameters } \\ \text { NAME } & \text { H22442 } \\ \text { EXPNO } & 1 \\ \text { PROCNO } & 1\end{array}$

F2 - Acquisition Parameters

Date_ 20040727

$\begin{array}{lr}\text { Date } & 20040727 \\ \text { Time } & 8.40\end{array}$

ARX400

PULPROG 5 M Multin

$\begin{array}{lr}\text { PULPROG } & 29 \\ \text { TD } & 32768\end{array}$

SOLVENT

DS

0
SWH $\quad 6410.256 \mathrm{~Hz}$

FIDRES $\quad 0.195625 \mathrm{~Hz}$

AQ $\quad 2.5559540 \mathrm{sec}$

$\begin{array}{lr}1430 \\ \text { DW } & 78.000 \text { usec }\end{array}$

DE 111.43 use

$\begin{array}{lr}\text { TE } & 300.0 \mathrm{~K} \\ \text { D1 } & 2.00000000 \mathrm{sec}\end{array}$

$\begin{array}{rr}2.00000000 \mathrm{sec} \\ 01 & 2.00 \mathrm{usec} \\ 01 & 111.00 \mathrm{usz}\end{array}$

DE $\quad 111.43$ use

$\begin{array}{lr}\text { SF01 } & 400.1320000 \mathrm{MHz} \\ \text { NUCLEUS } & 1 \mathrm{H}\end{array}$

F2 - Processing parameters

SI 16384

$\quad 400.1300085 \mathrm{MHz}$

$\begin{array}{lc}\text { WDW } & \text { no } \\ \text { SSB } & 0 \\ \text { LB } & 0.00 \mathrm{~Hz}\end{array}$

$\begin{array}{lr}\text { LB } & 0.00 \mathrm{H} \\ \text { GB } & 0 \\ \text { PC } & 0.50\end{array}$

D NMR plot parameters

$\begin{array}{ll}\text { CX } & 20.00 \mathrm{~cm} \\ \text { Fip } & 12.000 \mathrm{cpm}\end{array}$

$\begin{array}{ll}\text { F1 } & 12.000 \mathrm{ppm} \\ \text { Fi } & 4801.56 \mathrm{~Hz}\end{array}$

$\begin{array}{ll}\text { F2P } & -0.500 \mathrm{ppm} \\ \mathrm{F} 2 & -200.07 \mathrm{~Hz}\end{array}$

PPMCM $\quad 0.62500 \mathrm{ppm} / \mathrm{c}$

$\begin{array}{lr}\mathrm{HZCM} & 0.62500 \mathrm{ppm} / \mathrm{cm} \\ & 250.08125 \mathrm{~Hz} / \mathrm{cm}\end{array}$

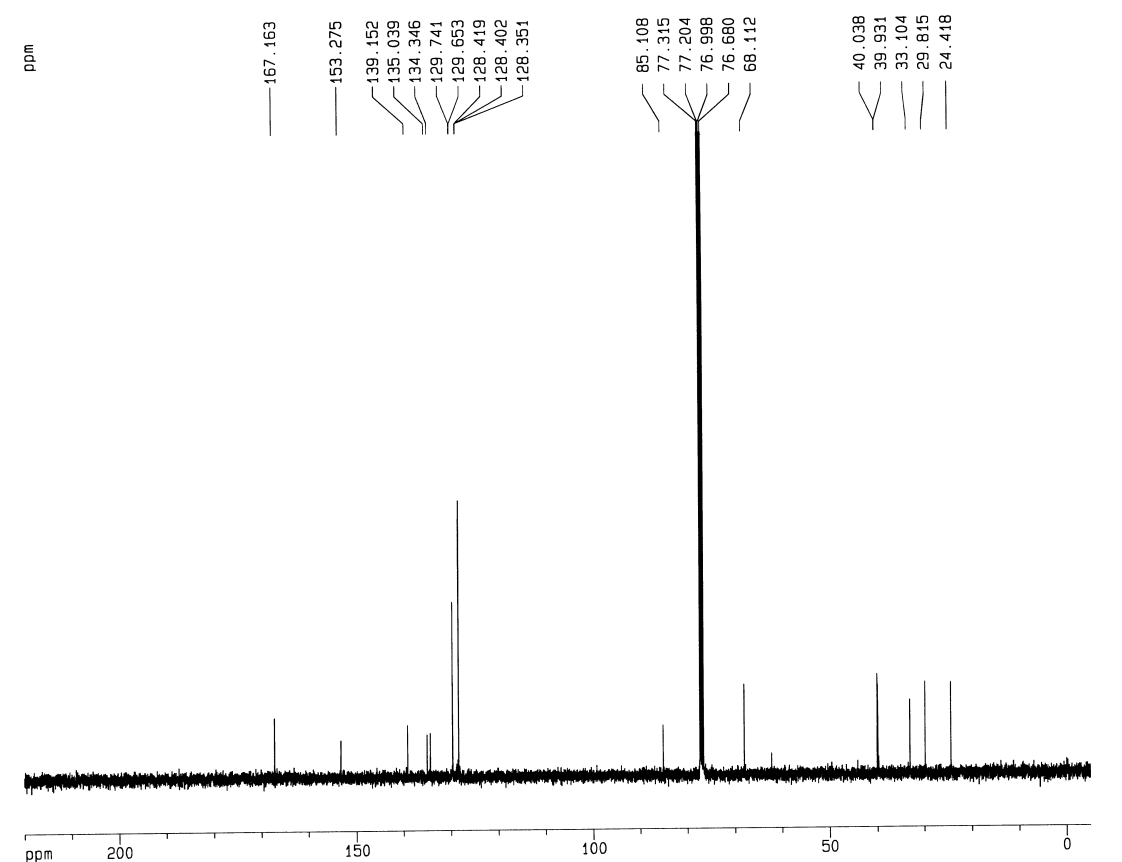

$\begin{array}{lr}\text { Current Data Parameters } \\ \text { NAME } & \text { C22442 } \\ \text { EXPNO } & 2 \\ \text { PROCNO } & 1\end{array}$

F2 - Acquisition Parameters

Date_ 20040727

$\begin{array}{lr}\text { Time } & 10.49 \\ \text { Time } & \text { PBX }\end{array}$

$5 \mathrm{~mm}$ Multinu

PUdC
PUPOG
T2768

$\begin{array}{ll}\text { TD } & 32760 \\ \text { SOLVENT } & \text { COC1 }\end{array}$

$\begin{array}{lc}\text { DS } & 400 \\ \text { SWH } & 0 \\ \text { P } & 29411.766 \mathrm{~Hz}\end{array}$

$29411.766 \mathrm{~Hz}$
IDRES $\quad 0.897576 \mathrm{~Hz}$

$\begin{array}{lc}\text { AQ } & 0.5571060 \mathrm{sec} \\ \text { PG } & 32768\end{array}$

17.000 use
DW
DE
24.29 use

$\begin{array}{ll}\text { TE } & 300.0 \mathrm{~K} \\ \text { TE } & 0.00002000 \mathrm{sec}\end{array}$

$\begin{array}{rr}D 12 & 0.00002000 \mathrm{sec} \\ \text { DL5 } & 24.00 \mathrm{~dB}\end{array}$

$\begin{array}{lr}\text { P31 } & 100.00 \mathrm{usec} \\ 01 & 2.00000000 \mathrm{sec}\end{array}$

P1 4.00 usec

$\begin{array}{lr}24.29 \mathrm{usec} \\ \mathrm{DE} & 100.6240000 \mathrm{MHz}\end{array}$

DELUS $\quad 100.024000$

$0.03000000 \mathrm{sec}$

$\begin{array}{lc}\text { F2 - Processing parameters } \\ \text { SI } & 327768 \\ \text { SF } & 100.6127711 \mathrm{MHz} \\ \text { WDW } & \text { EM } \\ \text { SSB } & 0 \\ \text { LB } & 0.50 \mathrm{~Hz} \\ \text { GB } & 0 \\ \text { PC } & 0.30\end{array}$

10 NMR plot parameters

$\begin{array}{lr}\text { CX } & 20.00 \mathrm{~cm} \\ \text { F1P } & 220.000 \mathrm{ppm}\end{array}$

$\begin{array}{lr}\text { F1P } & 220.000 \mathrm{ppm} \\ \text { F1 } & 22134.81 \mathrm{~Hz}\end{array}$

$\begin{array}{ll}-5.000 \mathrm{ppm} \\ \mathrm{FP}\end{array}-5.03 .07 \mathrm{pm}$

$\begin{array}{ll}\text { F2 } & -503.07 \mathrm{~Hz} \\ \text { PPMCM } & 11.25000 \mathrm{PDz} / \mathrm{c}\end{array}$ 
10a<smiles>O=C1NCC2C(=O)N3c4ccccc4Oc4ccccc4C3N(c3ccccc3Oc3ccccc32)c2ccccc21</smiles>

$$
\begin{aligned}
& \begin{array}{l}
\text { JL118-2 } \\
\operatorname{exp1} \quad \text { s2put }
\end{array}
\end{aligned}
$$

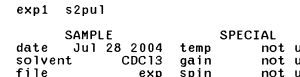

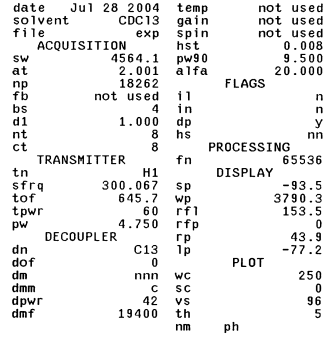
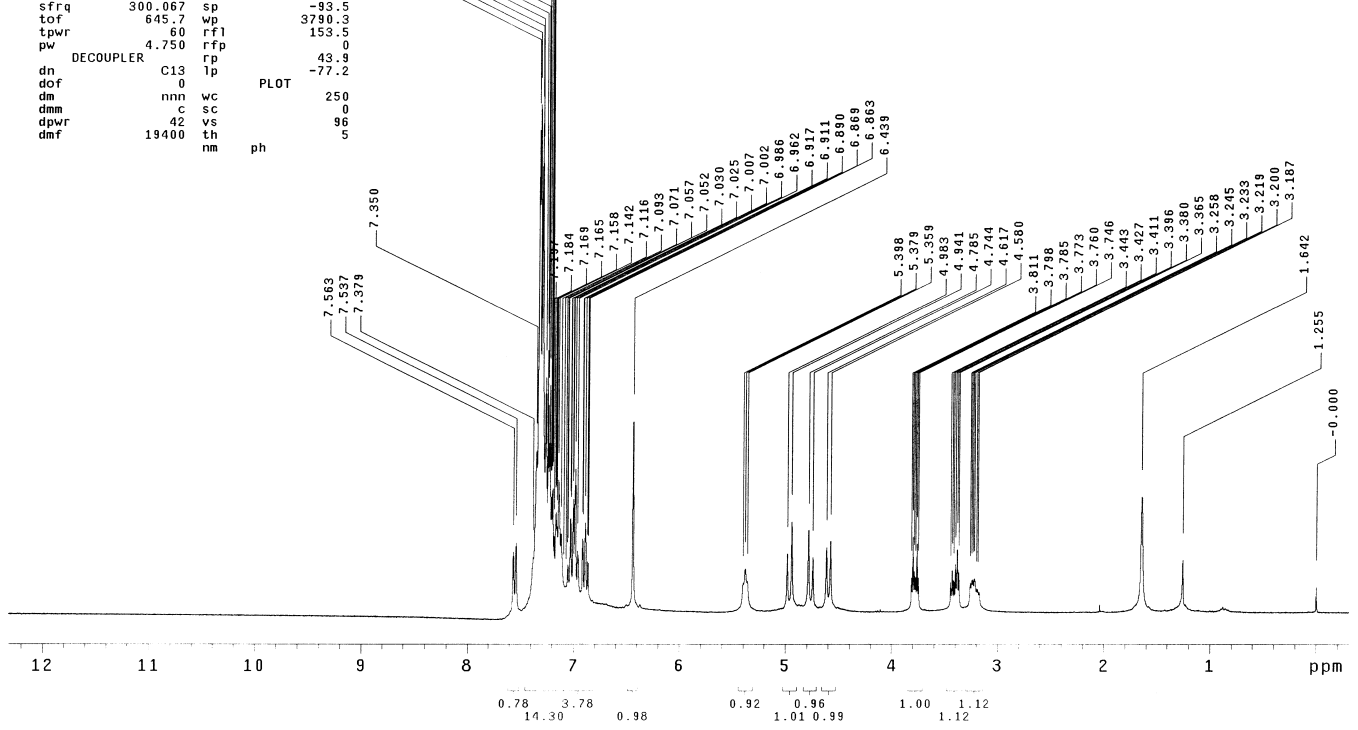

JL118-2

exp2 std13c

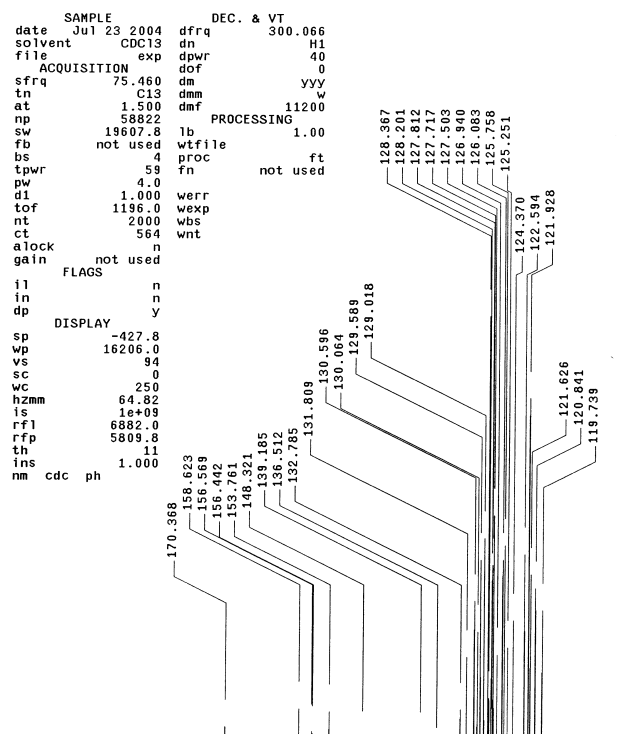

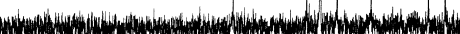




\section{DEPT of 10a}

JL118-2

Pulse Sequence: dept

CH3 carbons

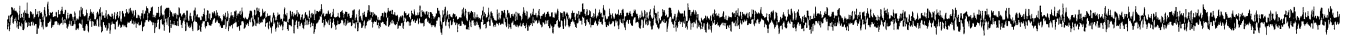

CH2 carbons

17 .

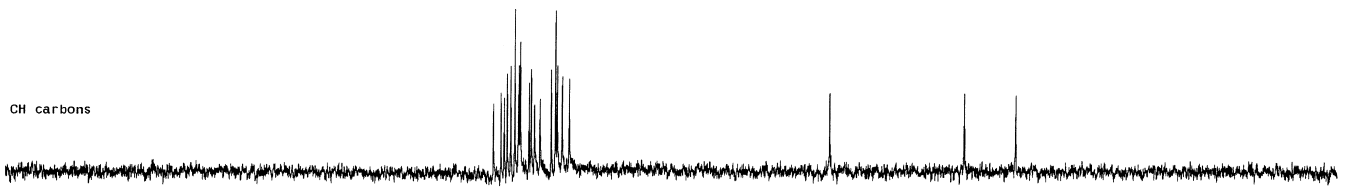

a11 protonated carbons

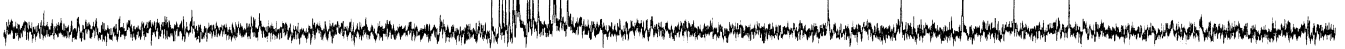

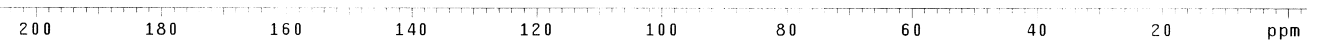


10b<smiles>Cc1ccc2c(c1)C1C(CNC(=O)OCc3ccccc3)C(=O)N3c4ccccc4Oc4ccc(C)cc4C3N1c1ccccc1O2</smiles>

JL134-2

exp1 s2pu1

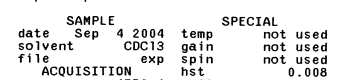

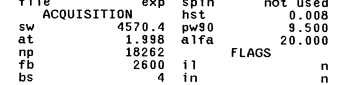

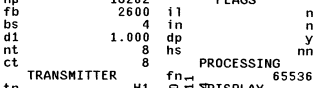

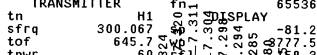

$\substack{\text { towr } \\ \text { pwo }}$

DECOUPLER ${ }_{\text {C13 }}^{4.750} \underset{\mathrm{rap}}{\mathrm{rap}}$

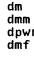

$\begin{array}{rl}0 & W C \\ n n n & w C \\ c & 5 C \\ 42 & v s \\ 19400 & \text { th } \\ & n m\end{array}$
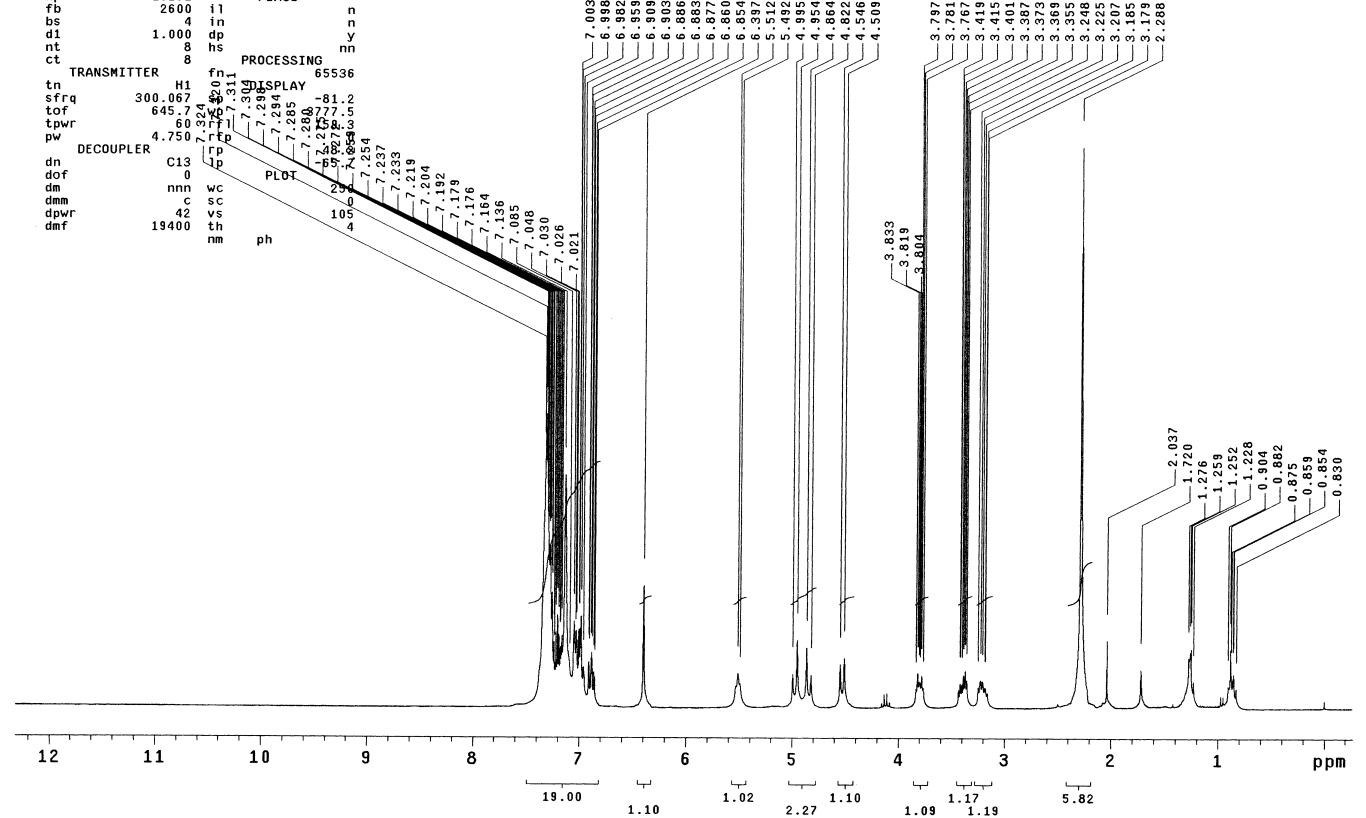

JL134-2

exp2 std13c

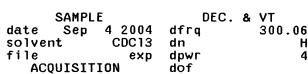

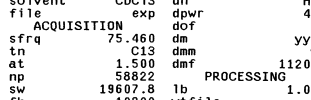

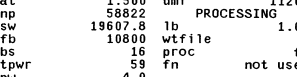

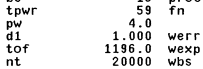

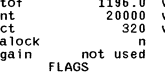

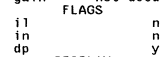

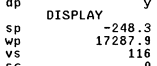

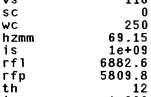

nns
nm no ph 1.000

$$
\text { (1) }
$$

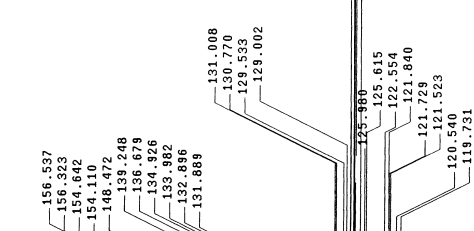

$$
\text { }
$$

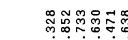


11<smiles>O=C(NCCC(=O)N1CCCSC1=Cc1ccccc1)OCc1ccccc1</smiles>

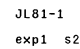

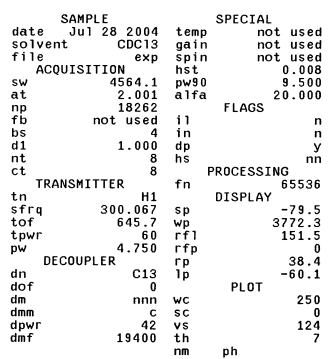

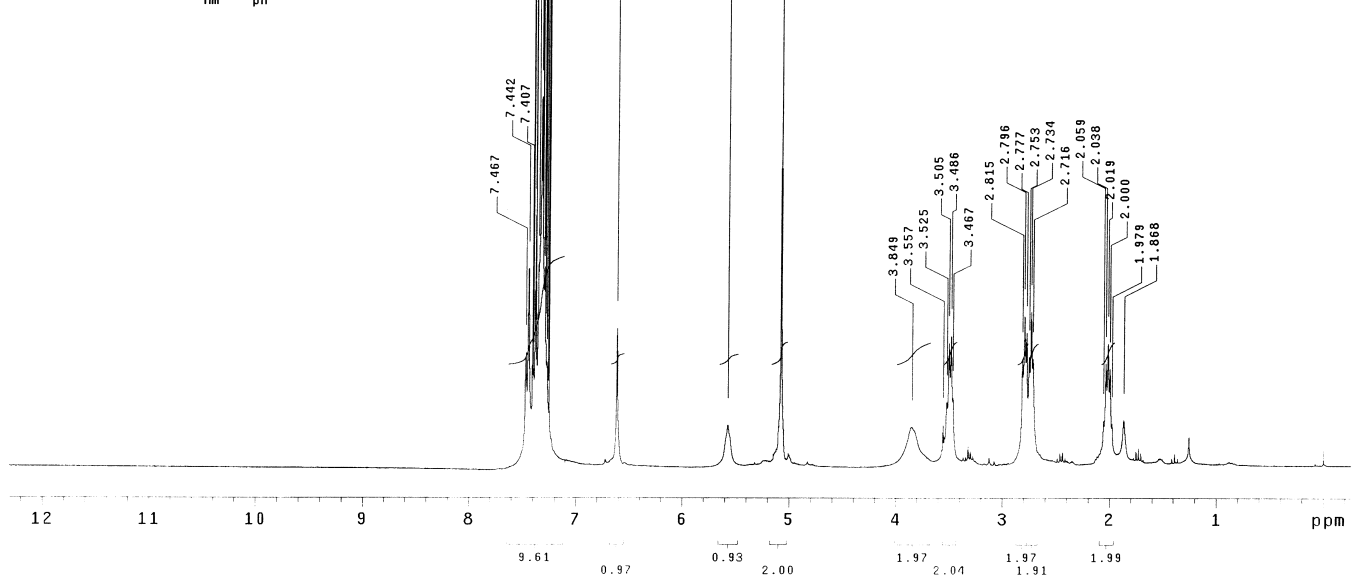

JL81-1
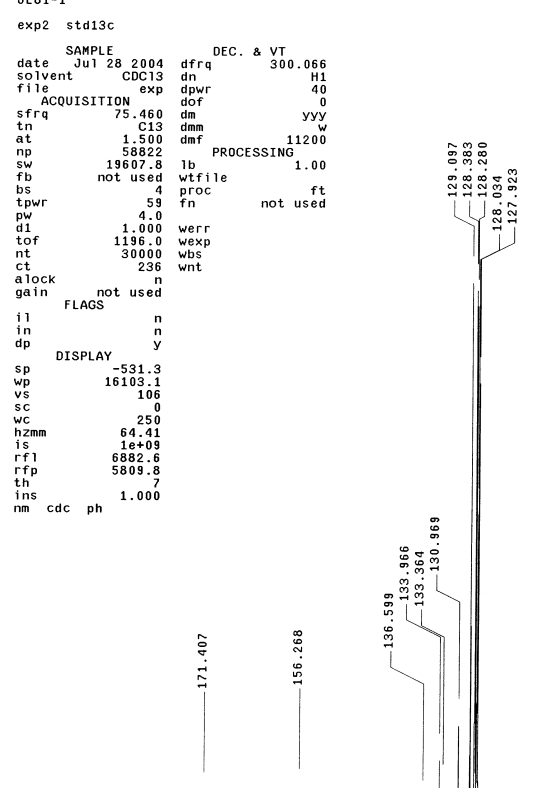


\section{Crystal Structure of $6 a^{6}$ (ORTEP Figure)}

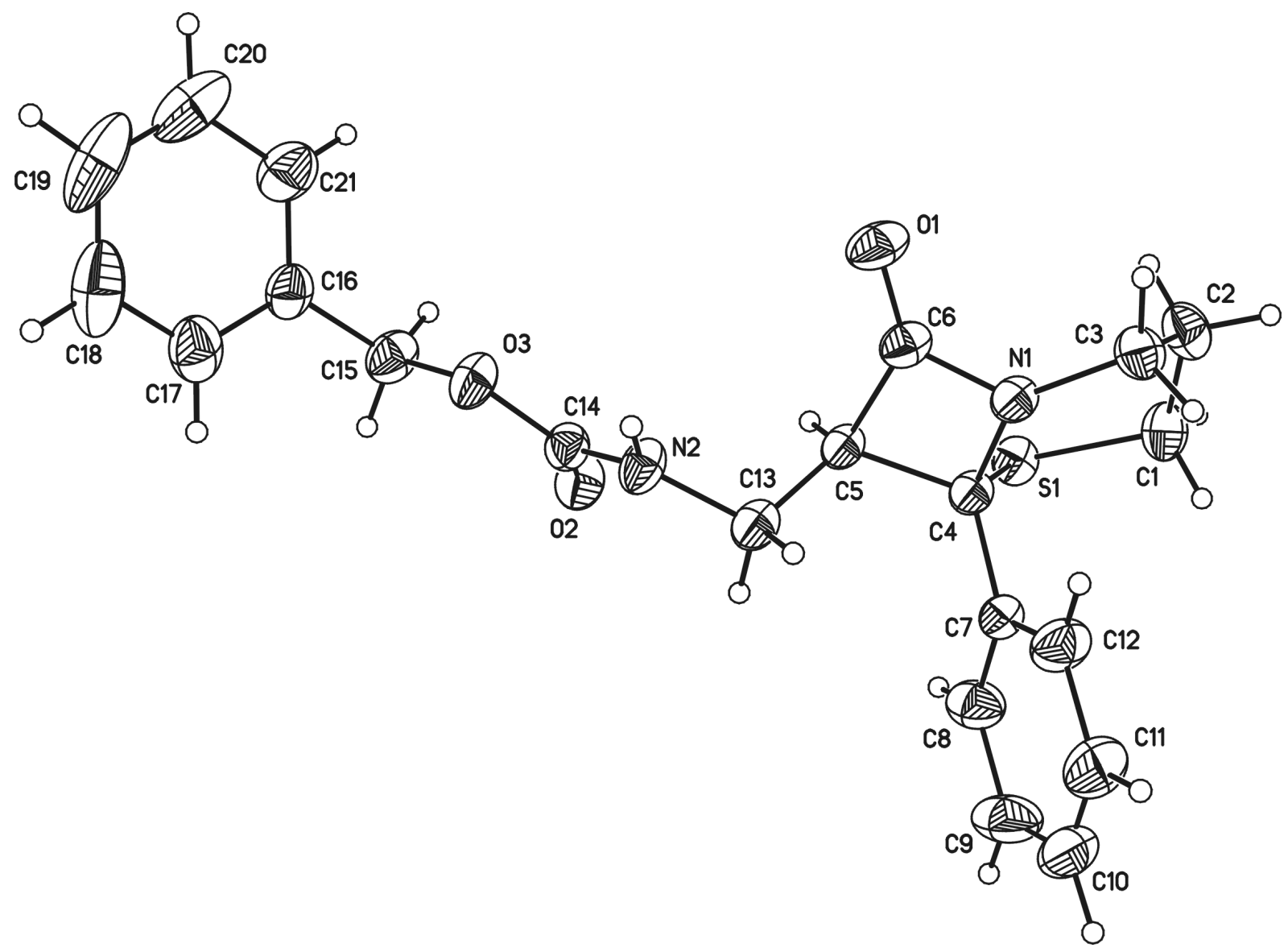

${ }^{6}$ CCDC 246471 contains the supplementary crystallographic data for this paper. These data can be obtained free of charge via www.ccdc.cam.ac.uk/data_request/cif, by emailing data_request@ @ccdc.cam.ac.uk, or by contacting The Cambridge Crystallographic Data Centre, 12, Union Road, Cambridge CB2 1EZ, UK; fax: +44 1223336033. 